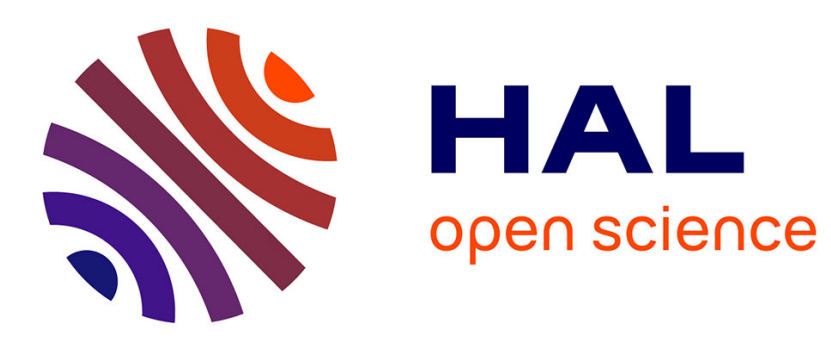

\title{
BCARET Model Checking for Malware Detection.
}

Huu Vu Nguyen, Tayssir Touili

\section{To cite this version:}

Huu Vu Nguyen, Tayssir Touili. BCARET Model Checking for Malware Detection.. Theoretical Aspects of Computing - ICTAC 2019 - 16th International Colloquium, Hammamet, Tunisia, October 31 - November 4, 2019, Proceedings., Oct 2019, Hammamet, Tunisia. hal-02389564

\section{HAL Id: hal-02389564 https://hal.science/hal-02389564}

Submitted on 1 Dec 2020

HAL is a multi-disciplinary open access archive for the deposit and dissemination of scientific research documents, whether they are published or not. The documents may come from teaching and research institutions in France or abroad, or from public or private research centers.
L'archive ouverte pluridisciplinaire HAL, est destinée au dépôt et à la diffusion de documents scientifiques de niveau recherche, publiés ou non, émanant des établissements d'enseignement et de recherche français ou étrangers, des laboratoires publics ou privés. 


\title{
BCARET Model Checking for Malware Detection
}

\author{
Huu-Vu Nguyen ${ }^{1}$, Tayssir Touili ${ }^{2}$ \\ ${ }^{1}$ LIPN and University Paris 13, France \\ ${ }^{2}$ CNRS, LIPN and University Paris 13, France
}

\begin{abstract}
The number of malware is growing fast recently. Traditional malware detectors based on signature matching and code emulation are easy to bypass. To overcome this problem, model-checking appears as an efficient approach that has been extensively applied for malware detection in recent years. Pushdown systems were proposed as a natural model for programs, as they allow to take into account the program's stack into the model. CARET and BCARET were proposed as formalisms for malicious behavior specification since they can specify properties that require matchings of calls and returns which is crucial for malware detection. In this paper, we propose to use BCARET for malicious behavior specification. Since BCARET formulas for malicious behaviors are huge, we propose to extend BCARET with variables, quantifiers and predicates over the stack. Our new logic is called SBPCARET. We reduce the malware detection problem to the model checking problem of PDSs against SBPCARET formulas, and we propose an efficient algorithm to model check SBPCARET formulas for PDSs.
\end{abstract}

\section{Introduction}

The number of malware is growing fast in recent years. Traditional approaches including signature matching and code emulation are not efficient enough to detect malwares. While attackers can use obfuscation techniques to hide their malware from the signature based malware detectors easily, the code emulation approaches can only track programs in certain execution paths due to the limited execution time. To overcome these limitations, model-checking appears as an efficient approach for malware detection, since model-checking allows to check the behaviors of a program in all its execution traces without executing it.

A lof of works have been investigated to apply model-checking for malware detection $[2,4,3,12,11,10,7]$. [4] proposed to use finite state graphs to model the program and use the temporal logic CTPL to specify malicious behaviours. However, finite graphs are not exact enough to model programs, as they don't allow to take into account the program's stack into the model. Indeed, the program's stack is usually used by malware writers for code obfuscation as explained in [5]. In addition, in binary codes and assembly programs, parameters are passed to functions by pushing them on the stack before the call is made. The values of these parameters are used to determine whether the program is malicious or 
not [6]. Therefore, being able to record the program's stack is critical for malware detection. To this aim, $[12,11,10,13]$ proposed to use pushdown systems to model programs, and defined extensions of LTL and CTL (called SLTPL and SCTPL) to precisely and compactly describe malicious behaviors. However, these logics cannot specify properties that require matchings of calls and returns, which is crucial to describe malicious behaviours [8]. Let us consider the typical behaviour of a spyware to illustrate this. The typical behaviour of a spyware is seeking personal information (emails, bank account information,...) on local drives by searching files that match specific conditions. To do that, it has to search directories of the host to look for interesting files whose names match a certain condition. If a file is found, the spyware will invoke a payload to steal the information, then continue looking for the remaining matching files. If a folder is found, it will pass into the folder path and continue investigating the folder recursively. To obtain this behavior, the spyware first calls the API FindFirstFile A to search for the first matching file in a given folder path. After that, it has to check whether the call to the API function FindFirstFileA is successful or not. When the function call fails, the spyware will call the API GetLastError. Otherwise, when the function call succeeds, a search handle $h$ will be returned by FindFirstFileA. There are two possibilities in this case. If the returned result is a folder, it will call the API function FindFirstFileA again to search for matching results in the found folder. If the returned result is a file, it will call the function FindNextFile A using $h$ as first parameter to look for the remaining matching files. This behavior cannot be described by LTL or CTL since it requires to express that the return value of the API function FindFirstFileA should be used as input to the function FindNextFileA.

CARET was introduced to express linear-temporal properties that involve matchings of calls and returns [1] and CARET model-checking for PDSs was considered $[7,6]$. However, the above behaviour cannot be described by CARET since it is a branching-time property. To specify that behaviour naturally and intuitively, BCARET was introduced to express these branching-time properties that involve matchings of calls and returns [8]. Using BCARET, the above behavior can be expressed by the following formula:

$$
\begin{array}{r}
\varphi_{s b}=\bigvee_{d \in D} E F^{g}\left(\operatorname{call}(\text { FindFirstFileA }) \wedge E X^{a}(\text { eax }=d) \wedge A F^{a}\right. \\
(\operatorname{call}(\text { GetLastError }) \vee \operatorname{call}(\text { FindFirstFileA }) \\
\left.\left.\vee\left(\operatorname{call}(\text { FindNextFileA }) \wedge d \Gamma^{*}\right)\right)\right)
\end{array}
$$

where the $\bigvee$ is taken over all possible memory addresses $d$ that contain the values of search handles $h$ in the program, $E X^{a}$ is a BCARET operator saying that "next in some run, in the same procedural context"; $E F^{g}$ is the standard CTL EF operator (eventually in some run), while $A F^{a}$ is a BCARET operator stating that "eventually in all runs, in the same procedural context".

In binary codes and assembly programs, the return value of an API function is placed in the register eax. Therefore, the return value of FindFirstFileA is the value of the register eax at the corresponding return-point of the call. 
Then, the subformula (call(FindFirstFileA) $\wedge E X^{a}(e a x=d)$ ) expresses that there is a call to the API function FindFirstFileA whose return value is $d$ (the abstract successor of a call is its corresponding return-point). A call to FindNextFileA requires a search handle $h$ as parameter and $h$ must be put on top of the program's stack (as parameters are passed through the stack in assembly programs). To express that $d$ is on top of the program stack, we use the regular expression $d \Gamma^{*}$. Thus, the subformula [call(FindNextFileA) $\left.\wedge d \Gamma^{*}\right]$ states that the API FindNextFileA is invoked with $d$ as parameter ( $d$ stores the information of the search handle $h$ ). Therefore, $\varphi_{s b}$ states that there is a call to the function FindFirstFileA whose return value is $d$ (the search handle), then, in all runs starting from that call, there will be either a call to the API GetLastError or a call to the API function FindFirstFileA or a call to the function FindNextFileA in which $d$ is used as a parameter.

However, it can be seen that this formula is huge, since it considers the disjunction (of different BCARET formulas) over all possible memory addresses $d$ which contain the information of search handles $h$ in the program. To represent it in a more compact fashion, we follow the idea of $[4,12,10,6]$ and extend BCARET with variables, quantifiers, and predicates over the stack. We call our new logic SBPCARET. The above formula can be concisely described by a SBPCARET formula as follows:

$$
\begin{aligned}
& \varphi_{s b}^{\prime}=\exists \boldsymbol{x} E F^{g}\left(\operatorname{call}(\text { FindFirstFile } A) \wedge E X^{a}(\text { eax }=x) \wedge A F^{a}\right. \\
& (\text { call(GetLastError }) \vee \operatorname{call}(\text { FindFirstFileA) } \\
& \left.\left.\vee\left(\operatorname{call}(\text { FindNextFileA }) \wedge x \Gamma^{*}\right)\right)\right)
\end{aligned}
$$

Thus, we propose in this work to use pushdown systems (PDSs) to model programs, and SBPCARET formulas to specify malicious behaviors. We reduce the malware detection problem to the model checking problem of PDSs against SBPCARET formulas, and we propose an efficient algorithm to check whether a PDS satisfies a SBPCARET formula. Our algorithm is based on a reduction to the emptiness problem of Symbolic Alternating Büchi Pushdown Systems. This latter problem is already solved in [10].

The rest of paper is organized as follows. In Section 2, we recall the definitions of Pushdown Systems. Section 3 introduces our logic SBPCARET. Model checking SBPCARET for PDSs is presented in Section 4. Finally, we conclude in Section 5.

\section{Pushdown Systems: A model for sequential programs}

Pushdown systems is a natural model that was extensively used to model sequential programs. Translations from sequential programs to PDSs can be found e.g. in [9]. As will be discussed in the next section, to precisely describe malicious behaviors as well as context-related properties, we need to keep track of the call 
and return actions in each path. Thus, as done in [8], we adapt the PDS model in order to record whether a rule of a PDS corresponds to a call, a return, or another instruction. We call this model a Labelled Pushdown System. We also extend the notion of run in order to take into account matching returns of calls.

Definition 1. A Labelled Pushdown System (PDS) $\mathcal{P}$ is a tuple $(P, \Gamma, \Delta, \sharp)$, where $P$ is a finite set of control locations, $\Gamma$ is a finite set of stack alphabet, $\sharp \notin \Gamma$ is a bottom stack symbol and $\Delta$ is a finite subset of $((P \times \Gamma) \times(P \times$ $\left.\Gamma^{*}\right) \times\{$ call, ret, int $\left.\}\right)$. If $((p, \gamma),(q, \omega), t) \in \Delta(t \in\{$ call, ret, int $\})$, we also write $\langle p, \gamma\rangle \stackrel{t}{\rightarrow}\langle q, \omega\rangle \in \Delta$. Rules of $\Delta$ are of the following form, where $p \in P, q \in$ $P, \gamma, \gamma_{1}, \gamma_{2} \in \Gamma$, and $\omega \in \Gamma^{*}$ :

$-\left(r_{1}\right):\langle p, \gamma\rangle \stackrel{\text { call }}{\longrightarrow}\left\langle q, \gamma_{1} \gamma_{2}\right\rangle$

$-\left(r_{2}\right):\langle p, \gamma\rangle \stackrel{\text { ret }}{\longrightarrow}\langle q, \epsilon\rangle$

$-\left(r_{3}\right):\langle p, \gamma\rangle \stackrel{\text { int }}{\longrightarrow}\langle q, \omega\rangle$

Intuitively, a rule of the form $\langle p, \gamma\rangle \stackrel{\text { call }}{\longrightarrow}\left\langle q, \gamma_{1} \gamma_{2}\right\rangle$ corresponds to a call statement. Such a rule usually models a statement of the form $\gamma \stackrel{\text { call } \quad \text { proc }}{\longrightarrow} \gamma_{2}$. In this rule, $\gamma$ is the control point of the program where the function call is made, $\gamma_{1}$ is the entry point of the called procedure, and $\gamma_{2}$ is the return point of the call. A rule $r_{2}$ models a return, whereas a rule $r_{3}$ corresponds to a simple statement (neither a call nor a return). A configuration of $\mathcal{P}$ is a pair $\langle p, \omega\rangle$, where $p$ is a control location and $\omega \in \Gamma^{*}$ is the stack content. For technical reasons, we suppose w.l.o.g. that the bottom stack symbol $\sharp$ is never popped from the stack, i.e., there is no rule in the form $\langle p, \sharp\rangle \stackrel{t}{\rightarrow}\langle q, \omega\rangle \in \Delta(t \in\{$ call, ret, int $\})$. $\mathcal{P}$ defines a transition relation $\Rightarrow_{\mathcal{P}}(t \in\{$ call, ret, int $\})$ as follows: If $\langle p, \gamma\rangle \stackrel{t}{\rightarrow}\langle q, \omega\rangle$, then for every $\omega^{\prime} \in \Gamma^{*},\left\langle p, \gamma \omega^{\prime}\right\rangle \Rightarrow_{\mathcal{P}}\left\langle q, \omega \omega^{\prime}\right\rangle$. In other words, $\left\langle q, \omega \omega^{\prime}\right\rangle$ is an immediate successor of $\left\langle p, \gamma \omega^{\prime}\right\rangle$. Let $\stackrel{*}{\Rightarrow} \mathcal{P}$ be the reflexive and transitive closure of $\Rightarrow_{\mathcal{P}}$.

A run of $\mathcal{P}$ from $\left\langle p_{0}, \omega_{0}\right\rangle$ is a sequence $\left\langle p_{0}, \omega_{0}\right\rangle\left\langle p_{1}, \omega_{1}\right\rangle\left\langle p_{2}, \omega_{2}\right\rangle \ldots$ where $\left\langle p_{i}, \omega_{i}\right\rangle \in$ $P \times \Gamma^{*}$ s.t. for every $i \geq 0,\left\langle p_{i}, \omega_{i}\right\rangle \Rightarrow_{\mathcal{P}}\left\langle p_{i+1}, \omega_{i+1}\right\rangle$. Given a configuration $\langle p, \omega\rangle$, let $\operatorname{Traces}(\langle p, \omega\rangle)$ be the set of all possible runs starting from $\langle p, \omega\rangle$.

\subsection{Global and abstract successors}

Let $\pi=\left\langle p_{0}, \omega_{0}\right\rangle\left\langle p_{1}, \omega_{1}\right\rangle \ldots$ be a run starting from $\left\langle p_{0}, \omega_{0}\right\rangle$. Over $\pi$, two kinds of successors are defined for every position $\left\langle p_{i}, \omega_{i}\right\rangle$ :

- global-successor: The global-successor of $\left\langle p_{i}, \omega_{i}\right\rangle$ is $\left\langle p_{i+1}, \omega_{i+1}\right\rangle$ where $\left\langle p_{i+1}, \omega_{i+1}\right\rangle$ is an immediate successor of $\left\langle p_{i}, \omega_{i}\right\rangle$.

- abstract-successor: The abstract-successor of $\left\langle p_{i}, \omega_{i}\right\rangle$ is determined as follows:

- If $\left\langle p_{i}, \omega_{i}\right\rangle \Rightarrow_{\mathcal{P}}\left\langle p_{i+1}, \omega_{i+1}\right\rangle$ corresponds to a call statement, there are two cases: (1) if $\left\langle p_{i}, \omega_{i}\right\rangle$ has $\left\langle p_{k}, \omega_{k}\right\rangle$ as a corresponding return-point in $\pi$, then, the abstract successor of $\left\langle p_{i}, \omega_{i}\right\rangle$ is $\left\langle p_{k}, \omega_{k}\right\rangle$; (2) if $\left\langle p_{i}, \omega_{i}\right\rangle$ does not have any corresponding return-point in $\pi$, then, the abstract successor of $\left\langle p_{i}, \omega_{i}\right\rangle$ is $\perp$.

- If $\left\langle p_{i}, \omega_{i}\right\rangle \Rightarrow_{\mathcal{P}}\left\langle p_{i+1}, \omega_{i+1}\right\rangle$ corresponds to a simple statement, the abstract successor of $\left\langle p_{i}, \omega_{i}\right\rangle$ is $\left\langle p_{i+1}, \omega_{i+1}\right\rangle$.

- If $\left\langle p_{i}, \omega_{i}\right\rangle \Rightarrow_{\mathcal{P}}\left\langle p_{i+1}, \omega_{i+1}\right\rangle$ corresponds to a return statement, the abstract successor of $\left\langle p_{i}, \omega_{i}\right\rangle$ is defined as $\perp$. 


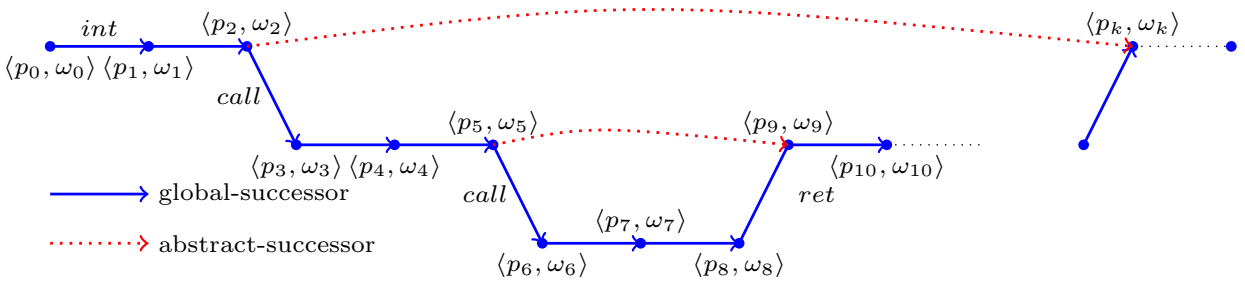

Fig. 1: Two kinds of successors on a run

For example, in Figure 1:

- The global-successors of $\left\langle p_{1}, \omega_{1}\right\rangle$ and $\left\langle p_{2}, \omega_{2}\right\rangle$ are $\left\langle p_{2}, \omega_{2}\right\rangle$ and $\left\langle p_{3}, \omega_{3}\right\rangle$ respectively.

- The abstract-successors of $\left\langle p_{2}, \omega_{2}\right\rangle$ and $\left\langle p_{5}, \omega_{5}\right\rangle$ are $\left\langle p_{k}, \omega_{k}\right\rangle$ and $\left\langle p_{9}, \omega_{9}\right\rangle$ respectively.

Let $\langle p, \omega\rangle$ be a configuration of a PDS $\mathcal{P}$. A configuration $\left\langle p^{\prime}, \omega^{\prime}\right\rangle$ is defined as a global-successor of $\langle p, \omega\rangle$ iff $\left\langle p^{\prime}, \omega^{\prime}\right\rangle$ is a global-successor of $\langle p, \omega\rangle$ over a run $\pi \in \operatorname{Traces}(\langle p, \omega\rangle)$. Similarly, a configuration $\left\langle p^{\prime}, \omega^{\prime}\right\rangle$ is defined as an abstractsuccessor of $\langle p, \omega\rangle$ iff $\left\langle p^{\prime}, \omega^{\prime}\right\rangle$ is an abstract-successor of $\langle p, \omega\rangle$ over a run $\pi \in$ $\operatorname{Traces}(\langle p, \omega\rangle)$

A global-path of $\mathcal{P}$ from $\left\langle p_{0}, \omega_{0}\right\rangle$ is a sequence $\left\langle p_{0}, \omega_{0}\right\rangle\left\langle p_{1}, \omega_{1}\right\rangle\left\langle p_{2}, \omega_{2}\right\rangle \ldots$ where $\left\langle p_{i}, \omega_{i}\right\rangle \in P \times \Gamma^{*}$ s.t. for every $i \geq 0,\left\langle p_{i+1}, \omega_{i+1}\right\rangle$ is a global-successor of $\left\langle p_{i}, \omega_{i}\right\rangle$. Similarly, an abstract-path of $\mathcal{P}$ from $\left\langle p_{0}, \omega_{0}\right\rangle$ is a sequence $\left\langle p_{0}, \omega_{0}\right\rangle\left\langle p_{1}, \omega_{1}\right\rangle\left\langle p_{2}, \omega_{2}\right\rangle \ldots$ where $\left\langle p_{i}, \omega_{i}\right\rangle \in P \times \Gamma^{*}$ s.t. for every $i \geq 0,\left\langle p_{i+1}, \omega_{i+1}\right\rangle$ is an abstract-successor of $\left\langle p_{i}, \omega_{i}\right\rangle$. For instance, in Figure 1, $\left\langle p_{0}, \omega_{0}\right\rangle\left\langle p_{1}, \omega_{1}\right\rangle\left\langle p_{2}, \omega_{2}\right\rangle\left\langle p_{3}, \omega_{3}\right\rangle\left\langle p_{4}, \omega_{4}\right\rangle\left\langle p_{5}, \omega_{5}\right\rangle \ldots$ is a global-path, while $\left\langle p_{0}, \omega_{0}\right\rangle\left\langle p_{1}, \omega_{1}\right\rangle\left\langle p_{2}, \omega_{2}\right\rangle\left\langle p_{k}, \omega_{k}\right\rangle \ldots$ is an abstract-path.

\section{Malicious Behaviour Specification}

In this section, we define the Stack Branching temporal Predicate logic of CAlls and RETurns (SBPCARET) as an extension of BCARET [8] with variables and regular predicates over the stack contents. The predicates contain variables that can be quantified existentially or universally. Regular predicates are expressed by regular variable expressions and are used to describe the stack content of PDSs.

\subsection{Environments, Predicates and Regular Variable Expressions}

Let $\mathcal{X}=\left\{x_{1}, \ldots, x_{n}\right\}$ be a finite set of variables over a finite domain $\mathcal{D}$. Let $B: \mathcal{X} \cup \mathcal{D} \rightarrow \mathcal{D}$ be an environment that associates each variable $x \in \mathcal{X}$ with a value $d \in \mathcal{D}$ s.t $B(d)=d$ for every $d \in \mathcal{D}$. Let $B[x \leftarrow d]$ be an environment obtained from $B$ such that $B[x \leftarrow d](x)=d$ and $B[x \leftarrow d](y)=B(y)$ for every $y \neq x$. Let $A b s_{x}(B)=\left\{B^{\prime} \in \mathcal{B} \mid \forall y \in \mathcal{X}, y \neq x, B(y)=B^{\prime}(y)\right\}$ be the function that abstracts away the value of $x$. Let $\mathcal{B}$ be the set of all environments.

Let $A P=\{a, b, c, \ldots\}$ be a finite set of atomic propositions. Let $A P_{\mathcal{D}}$ be a finite set of atomic predicates of the form $b\left(\alpha_{1}, \ldots, \alpha_{m}\right)$ such that $b \in A P$ and 
$\alpha_{i} \in \mathcal{D}$ for every $1 \leq i \leq m$. Let $A P_{\mathcal{X}}$ be a finite set of atomic predicates $b\left(\alpha_{1}, \ldots, \alpha_{n}\right)$ such that $b \in A P$ and $\alpha_{i} \in \mathcal{X} \cup \mathcal{D}$ for every $1 \leq i \leq n$.

Let $\mathcal{P}=(P, \Gamma, \Delta)$ be a Labelled PDS. A Regular Variable Expression (RVE) $e$ over $\mathcal{X} \cup \Gamma$ is defined by $e::=\epsilon|a \in \mathcal{X} \cup \Gamma| e+e \mid$ e.e $\mid e^{*}$. The language $L(e)$ of a $\operatorname{RVE} e$ is a subset of $P \times \Gamma^{*} \times \mathcal{B}$ and is defined as follows:

- $L(\epsilon)=\{(\langle p, \epsilon\rangle, B) \mid p \in P, B \in \mathcal{B}\}$

- for $x \in \mathcal{X}, L(x)=\{(\langle p, \gamma\rangle, B) \mid p \in P, \gamma \in \Gamma, B \in \mathcal{B}$ s.t $B(x)=\gamma\}$

- for $\gamma \in \Gamma, L(\gamma)=\{(\langle p, \gamma\rangle, B) \mid p \in P, B \in \mathcal{B}\}$

- L $\left(e_{1} \cdot e_{2}\right)=\left\{\left(\left\langle p, \omega^{\prime} \omega^{\prime \prime}\right\rangle, B\right) \mid\left(\left\langle p, \omega^{\prime}\right\rangle, B\right) \in L\left(e_{1}\right) ;\left(\left\langle p, \omega^{\prime \prime}\right\rangle, B\right) \in L\left(e_{2}\right)\right\}$

- $L\left(e^{*}\right)=\left\{(\langle p, \omega\rangle, B) \mid \omega \in\left\{v \in \Gamma^{*} \mid(\langle p, v\rangle, B) \in L(e)\right\}^{*}\right\}$

\subsection{The Stack Branching temporal Predicate logic of CAlls and RETurns - SBPCARET}

A SBPCARET formula is a BCARET formula where predicates and RVEs are used as atomic propositions and where quantifiers are applied to variables. For technical reasons, we assume w.l.o.g. that formulas are written in positive normal form, where negations are applied only to atomic predicates, and we use the release operator $R$ as the dual of the until operator $U$. From now on, we fix a finite set of variables $\mathcal{X}$, a finite set of atomic propositions $A P$, a finite domain $\mathcal{D}$, and a finite set of RVEs $\mathcal{V}$. A SBPCARET formula is defined as follows, where $v \in\{g, a\}, x \in \mathcal{X}, e \in \mathcal{V}, b\left(\alpha_{1}, \ldots, \alpha_{n}\right) \in A P_{\mathcal{X}}$ :

$$
\begin{aligned}
\varphi:= & \text { true } \mid \text { false }\left|b\left(\alpha_{1}, \ldots, \alpha_{n}\right)\right| \neg b\left(\alpha_{1}, \ldots, \alpha_{n}\right)|e| \neg e|\varphi \vee \varphi| \varphi \wedge \varphi|\forall x \varphi| \\
& \exists x \varphi\left|E X^{v} \varphi\right| A X^{v} \varphi\left|E\left[\varphi U^{v} \varphi\right]\right| A\left[\varphi U^{v} \varphi\right]\left|E\left[\varphi R^{v} \varphi\right]\right| A\left[\varphi R^{v} \varphi\right]
\end{aligned}
$$

Let $\lambda: P \longrightarrow 2^{A P_{\mathcal{D}}}$ be a labelling function which associates each control location to a set of atomic predicates. Let $\varphi$ be a SBPCARET formula over $A P$. Let $\langle p, \omega\rangle$ be a configuration of $\mathcal{P}$. Then we say that $\mathcal{P}$ satisfies $\varphi$ at $\langle p, \omega\rangle$ (denoted by $\langle p, \omega\rangle \models_{\lambda} \varphi$ ) iff there exists an environment $B \in \mathcal{B}$ such that $\langle p, \omega\rangle$ satisfies $\varphi$ under $B$ (denoted by $\langle p, \omega\rangle \models_{\lambda}^{B} \varphi$ ). The satisfiability relation of a SBPCARET formula $\varphi$ at a configuration $\left\langle p_{0}, \omega_{0}\right\rangle$ under the environment $B$ w.r.t. the labelling function $\lambda$, denoted by $\left\langle p_{0}, \omega_{0}\right\rangle \vDash_{\lambda}^{B} \varphi$, is defined inductively as follows:

- $\left\langle p_{0}, \omega_{0}\right\rangle \vDash_{\lambda}^{B}$ true for every $\left\langle p_{0}, \omega_{0}\right\rangle$

- $\left\langle p_{0}, \omega_{0}\right\rangle \not \nvdash_{\lambda}^{B}$ false for every $\left\langle p_{0}, \omega_{0}\right\rangle$

$-\left\langle p_{0}, \omega_{0}\right\rangle \vDash_{\lambda}^{B} b\left(\alpha_{1}, \ldots, \alpha_{n}\right)$, iff $b\left(B\left(\alpha_{1}\right), \ldots, B\left(\alpha_{n}\right)\right) \in \lambda\left(p_{0}\right)$

$-\left\langle p_{0}, \omega_{0}\right\rangle \models_{\lambda}^{B} \neg b\left(\alpha_{1}, \ldots, \alpha_{n}\right)$, iff $b\left(B\left(\alpha_{1}\right), \ldots, B\left(\alpha_{n}\right)\right) \notin \lambda\left(p_{0}\right)$

$-\left\langle p_{0}, \omega_{0}\right\rangle \vDash{ }_{\lambda}^{B}$ e iff $\left(\left\langle p_{0}, \omega_{0}\right\rangle, B\right) \in L(e)$

$-\left\langle p_{0}, \omega_{0}\right\rangle \vDash_{\lambda}^{B} \neg e$ iff $\left(\left\langle p_{0}, \omega_{0}\right\rangle, B\right) \notin L(e)$

$-\left\langle p_{0}, \omega_{0}\right\rangle \vDash_{\lambda}^{B} \varphi_{1} \vee \varphi_{2}$ iff $\left(\left\langle p_{0}, \omega_{0}\right\rangle \vDash_{\lambda}^{B} \varphi_{1}\right.$ or $\left.\left\langle p_{0}, \omega_{0}\right\rangle \vDash_{\lambda}^{B} \varphi_{2}\right)$

$-\left\langle p_{0}, \omega_{0}\right\rangle \vDash_{\lambda}^{B} \varphi_{1} \wedge \varphi_{2}$ iff $\left(\left\langle p_{0}, \omega_{0}\right\rangle \models_{\lambda}^{B} \varphi_{1}\right.$ and $\left.\left\langle p_{0}, \omega_{0}\right\rangle \models_{\lambda}^{B} \varphi_{2}\right)$

$-\left\langle p_{0}, \omega_{0}\right\rangle \vDash_{\lambda}^{B} \forall x \varphi$ iff for every $d \in \mathcal{D},\left\langle p_{0}, \omega_{0}\right\rangle \vDash_{\lambda}^{B[x \leftarrow d]} \varphi$

- $\left\langle p_{0}, \omega_{0}\right\rangle \models_{\lambda}^{B} \exists x \varphi$ iff there exists $d \in \mathcal{D},\left\langle p_{0}, \omega_{0}\right\rangle \vDash_{\lambda}^{B[x \leftarrow d]} \varphi$

- $\left\langle p_{0}, \omega_{0}\right\rangle \models_{\lambda}^{B} E X^{g} \varphi$ iff there exists a global-successor $\left\langle p^{\prime}, \omega^{\prime}\right\rangle$ of $\left\langle p_{0}, \omega_{0}\right\rangle$ such that $\left\langle p^{\prime}, \omega^{\prime}\right\rangle \models_{\lambda}^{B} \varphi$

- $\left\langle p_{0}, \omega_{0}\right\rangle \vDash_{\lambda}^{B} A X^{g} \varphi$ iff $\left\langle p^{\prime}, \omega^{\prime}\right\rangle \vDash_{\lambda}^{B} \varphi$ for every global-successor $\left\langle p^{\prime}, \omega^{\prime}\right\rangle$ of $\left\langle p_{0}, \omega_{0}\right\rangle$ 
- $\left\langle p_{0}, \omega_{0}\right\rangle \vDash{ }_{\lambda}^{B} E\left[\varphi_{1} U^{g} \varphi_{2}\right]$ iff there exists a global-path $\pi=\left\langle p_{0}, \omega_{0}\right\rangle\left\langle p_{1}, \omega_{1}\right\rangle\left\langle p_{2}, \omega_{2}\right\rangle \ldots$ of $\mathcal{P}$ starting from $\left\langle p_{0}, \omega_{0}\right\rangle$ s.t. $\exists i \geq 0,\left\langle p_{i}, \omega_{i}\right\rangle \vDash_{\lambda}^{B} \varphi_{2}$ and for every $0 \leq j<i$, $\left\langle p_{j}, \omega_{j}\right\rangle \vDash_{\lambda}^{B} \varphi_{1}$

- $\left\langle p_{0}, \omega_{0}\right\rangle \vDash_{\lambda}^{B} A\left[\varphi_{1} U^{g} \varphi_{2}\right]$ iff for every global-path $\pi=\left\langle p_{0}, \omega_{0}\right\rangle\left\langle p_{1}, \omega_{1}\right\rangle\left\langle p_{2}, \omega_{2}\right\rangle \ldots$ of $\mathcal{P}$ starting from $\left\langle p_{0}, \omega_{0}\right\rangle, \exists i \geq 0,\left\langle p_{i}, \omega_{i}\right\rangle \vDash_{\lambda}^{B} \varphi_{2}$ and for every $0 \leq j<i$, $\left\langle p_{j}, \omega_{j}\right\rangle \vDash_{\lambda}^{B} \varphi_{1}$

- $\left\langle p_{0}, \omega_{0}\right\rangle \models_{\lambda}^{B} E\left[\varphi_{1} R^{g} \varphi_{2}\right]$ iff there exists a global-path $\pi=\left\langle p_{0}, \omega_{0}\right\rangle\left\langle p_{1}, \omega_{1}\right\rangle\left\langle p_{2}, \omega_{2}\right\rangle \ldots$ of $\mathcal{P}$ starting from $\left\langle p_{0}, \omega_{0}\right\rangle$ s.t. for every $i \geq 0$, if $\left\langle p_{i}, \omega_{i}\right\rangle \nvdash_{\lambda}^{B} \varphi_{2}$ then there exists $0 \leq j<i$ s.t. $\left\langle p_{j}, \omega_{j}\right\rangle \vDash_{\lambda}^{B} \varphi_{1}$

- $\left\langle p_{0}, \omega_{0}\right\rangle \vDash_{\lambda}^{B} A\left[\varphi_{1} R^{g} \varphi_{2}\right]$ iff for every global-path $\pi=\left\langle p_{0}, \omega_{0}\right\rangle\left\langle p_{1}, \omega_{1}\right\rangle\left\langle p_{2}, \omega_{2}\right\rangle \ldots$ of $\mathcal{P}$ starting from $\left\langle p_{0}, \omega_{0}\right\rangle$, for every $i \geq 0$, if $\left\langle p_{i}, \omega_{i}\right\rangle \not \nvdash_{\lambda}^{B} \varphi_{2}$ then there exists $0 \leq j<i$ s.t. $\left\langle p_{j}, \omega_{j}\right\rangle \vDash_{\lambda}^{B} \varphi_{1}$

- $\left\langle p_{0}, \omega_{0}\right\rangle \vDash_{\lambda}^{B} E X^{a} \varphi$ iff there exists an abstract-successor $\left\langle p^{\prime}, \omega^{\prime}\right\rangle$ of $\left\langle p_{0}, \omega_{0}\right\rangle$ such that $\left\langle p^{\prime}, \omega^{\prime}\right\rangle \vDash_{\lambda}^{B} \varphi$

- $\left\langle p_{0}, \omega_{0}\right\rangle \vDash_{\lambda}^{B} A X^{a} \varphi$ iff $\left\langle p^{\prime}, \omega^{\prime}\right\rangle \vDash_{\lambda}^{B} \varphi$ for every abstract-successor $\left\langle p^{\prime}, \omega^{\prime}\right\rangle$ of $\left\langle p_{0}, \omega_{0}\right\rangle$

- $\left\langle p_{0}, \omega_{0}\right\rangle \models_{\lambda}^{B} E\left[\varphi_{1} U^{a} \varphi_{2}\right]$ iff there exists an abstract-path $\pi=\left\langle p_{0}, \omega_{0}\right\rangle\left\langle p_{1}, \omega_{1}\right\rangle\left\langle p_{2}, \omega_{2}\right\rangle \ldots$ of $\mathcal{P}$ starting from $\left\langle p_{0}, \omega_{0}\right\rangle$ s.t. $\exists i \geq 0,\left\langle p_{i}, \omega_{i}\right\rangle \vDash_{\lambda}^{B} \varphi_{2}$ and for every $0 \leq j<i$, $\left\langle p_{j}, \omega_{j}\right\rangle \vDash_{\lambda}^{B} \varphi_{1}$

- $\left\langle p_{0}, \omega_{0}\right\rangle \vDash_{\lambda}^{B} A\left[\varphi_{1} U^{a} \varphi_{2}\right]$ iff for every abstract-path $\pi=\left\langle p_{0}, \omega_{0}\right\rangle\left\langle p_{1}, \omega_{1}\right\rangle\left\langle p_{2}, \omega_{2}\right\rangle \ldots$ of $\mathcal{P}, \exists i \geq 0,\left\langle p_{i}, \omega_{i}\right\rangle \vDash_{\lambda}^{B} \varphi_{2}$ and for every $0 \leq j<i,\left\langle p_{j}, \omega_{j}\right\rangle \vDash_{\lambda}^{B} \varphi_{1}$

- $\left\langle p_{0}, \omega_{0}\right\rangle \vDash_{\lambda}^{B} E\left[\varphi_{1} R^{a} \varphi_{2}\right]$ iff there exists an abstract-path $\pi=\left\langle p_{0}, \omega_{0}\right\rangle\left\langle p_{1}, \omega_{1}\right\rangle\left\langle p_{2}, \omega_{2}\right\rangle \ldots$ of $\mathcal{P}$ starting from $\left\langle p_{0}, \omega_{0}\right\rangle$ s.t. for every $i \geq 0$, if $\left\langle p_{i}, \omega_{i}\right\rangle \nvdash_{\lambda}^{B} \varphi_{2}$ then there exists $0 \leq j<i$ s.t. $\left\langle p_{j}, \omega_{j}\right\rangle \vDash_{\lambda}^{B} \varphi_{1}$

- $\left\langle p_{0}, \omega_{0}\right\rangle \models_{\lambda}^{B} A\left[\varphi_{1} R^{a} \varphi_{2}\right]$ iff for every abstract-path $\pi=\left\langle p_{0}, \omega_{0}\right\rangle\left\langle p_{1}, \omega_{1}\right\rangle\left\langle p_{2}, \omega_{2}\right\rangle \ldots$ of $\mathcal{P}$ starting from $\left\langle p_{0}, \omega_{0}\right\rangle$, for every $i \geq 0$, if $\left\langle p_{i}, \omega_{i}\right\rangle \not \nvdash_{\lambda}^{B} \varphi_{2}$ then there exists $0 \leq j<i$ s.t. $\left\langle p_{j}, \omega_{j}\right\rangle \vDash_{\lambda}^{B} \varphi_{1}$

Other SBPCARET operators can be expressed by the above operators: $E F^{g} \varphi=$ $E\left[\right.$ true $\left.U^{g} \varphi\right], E F^{a} \varphi=E\left[\right.$ true $\left.U^{a} \varphi\right], A F^{g} \varphi=A\left[\operatorname{true} U^{g} \varphi\right], A F^{a} \varphi=A\left[\operatorname{true} U^{a} \varphi\right], \ldots$

Closure. Given a SBPCARET formula $\varphi$, the closure $C l(\varphi)$ is the set of all subformulae of $\varphi$, including $\varphi$. Let $A P^{+}(\varphi)=\left\{b\left(\alpha_{1}, \ldots, \alpha_{n}\right) \in A P_{\mathcal{X}} \mid b\left(\alpha_{1}, \ldots, \alpha_{n}\right) \in\right.$ $C l(\varphi)\} ; A P^{-}(\varphi)=\left\{b\left(\alpha_{1}, \ldots, \alpha_{n}\right) \in A P_{\mathcal{X}} \mid \neg b\left(\alpha_{1}, \ldots, \alpha_{n}\right) \in C l(\varphi)\right\}, \operatorname{Reg}^{+}(\varphi)=$ $\{e \in \mathcal{V} \mid e \in C l(\varphi)\}, \operatorname{Reg}^{-}(\varphi)=\{e \in \mathcal{V} \mid \neg e \in C l(\varphi)\}$

\section{SBPCARET Model-Checking for Pushdown Systems}

In this section, we show how to do SBPCARET model-checking for PDSs. Let then $\mathcal{P}$ be a PDS, $\varphi$ be a SBPCARET formula, and $\mathcal{V}$ be the set of RVEs occuring in $\varphi$. We follow the idea of [10] and use Variable Automata to represent RVEs.

\subsection{Variable Automata}

Given a $\operatorname{PDS} \mathcal{P}=(P, \Gamma, \Delta)$ s.t. $\Gamma \subseteq \mathcal{D}$, a Variable Automaton (VA) [10] is a tuple $(Q, \Gamma, \delta, s, F)$, where $Q$ is a finite set of states, $\Gamma$ is the input alphabet, $s \in Q$ is an initial state; $F \subseteq Q$ is a finite set of accepting states; and $\delta$ is a finite set of transition rules of the form $p \stackrel{\alpha}{\rightarrow}\left\{q_{1}, \ldots, q_{n}\right\}$ where $\alpha$ can be $x, \neg x$, or $\gamma$, for any $x \in \mathcal{X}$ and $\gamma \in \Gamma$. 
Let $B \in \mathcal{B}$. A run of VA on a word $\gamma_{1}, \ldots, \gamma_{m}$ under $B$ is a tree of height $m$ whose root is labelled by the initial state $s$, and each node at depth $k$ labelled by a state $q$ has $h$ children labelled by $p_{1}, \ldots, p_{h}$ respectively, such that:

- either $q \stackrel{\gamma_{k}}{\longrightarrow}\left\{p_{1}, \ldots, p_{h}\right\} \in \delta$ and $\gamma_{k} \in \Gamma$;

- or $q \stackrel{x}{\rightarrow}\left\{p_{1}, \ldots, p_{h}\right\} \in \delta, x \in \mathcal{X}$ and $B(x)=\gamma_{k}$;

- or $q \stackrel{\neg x}{\longrightarrow}\left\{p_{1}, \ldots, p_{h}\right\} \in \delta, x \in \mathcal{X}$ and $B(x) \neq \gamma_{k}$.

A branch of the tree is accepting iff the leaf of the branch is an accepting state. A run is accepting iff all its branches are accepting. A word $\omega \in \Gamma^{*}$ is accepted by a VA under an environment $B \in \mathcal{B}$ iff the VA has an accepting run on the word $\omega$ under the environment $B$.

The language of a VA $M$, denoted by $L(M)$, is a subset of $\left(P \times \Gamma^{*}\right) \times \mathcal{B}$. $(\langle p, \omega\rangle, B) \in L(M)$ iff $M$ accepts the word $\omega$ under the environment $B$.

Theorem 1. [10] For every regular expression $e \in \mathcal{V}$, we can compute in polynomial time a Variable Automaton $M$ s.t. $L(M)=L(e)$.

Theorem 2. [10] VAs are closed under boolean operations.

\subsection{Symbolic Alternating Büchi Pushdown Systems (SABPDSs).}

Definition 2. A Symbolic Alternating Büchi Pushdown System (SABPDS) is a tuple $\mathcal{B P}=(P, \Gamma, \Delta, F)$, where $P$ is a set of control locations, $\Gamma \subseteq \mathcal{D}$ is stack alphabet, $F \subseteq P \times 2^{\mathcal{B}}$ is a set of accepting control locations and $\Delta$ is a finite set of transitions of the form $\langle p, \gamma\rangle \stackrel{\mathbb{R}}{\hookrightarrow}\left\{\left\langle p_{1}, \omega_{1}\right\rangle, \ldots,\left\langle p_{n}, \omega_{n}\right\rangle\right\}$ where $p \in P, \gamma \in \Gamma$, for every $1 \leq i \leq n: p_{i} \in P, \omega_{i} \in \Gamma^{*}$; and $\mathbb{R}:(\mathcal{B})^{n} \rightarrow 2^{\mathcal{B}}$ is a function that maps a tuple of environments $\left(B_{1}, \ldots, B_{n}\right)$ to a set of environments.

A configuration of a SABPDS $\mathcal{B P}$ is a tuple $\langle\llbracket p, B \rrbracket, \omega\rangle$, where $p \in P$ is the current control location, $B \in \mathcal{B}$ is an environment and $\omega \in \Gamma^{*}$ is the current stack content. Let $\langle p, \gamma\rangle \stackrel{\mathbb{R}}{\hookrightarrow}\left\{\left\langle p_{1}, \omega_{1}\right\rangle, \ldots,\left\langle p_{n}, \omega_{n}\right\rangle\right\}$ be a rule of $\Delta$, then, for every $\omega \in \Gamma^{*}, B, B_{1}, \ldots, B_{n} \in \mathcal{B}$, if $B \in \mathbb{R}\left(B_{1}, \ldots, B_{n}\right)$, then the configuration $\langle\llbracket p, B \rrbracket, \gamma \omega\rangle$ (resp. $\left.\left\{\left\langle\llbracket p_{1}, B_{1} \rrbracket, \omega_{1} \omega\right\rangle, \ldots,\left\langle\llbracket p_{n}, B_{n} \rrbracket, \omega_{n} \omega\right\rangle\right\}\right)$ is an immediate predecessor (resp. successor) of $\left\{\left\langle\llbracket p_{1}, B_{1} \rrbracket, \omega_{1} \omega\right\rangle, \ldots,\left\langle\llbracket p_{n}, B_{n} \rrbracket, \omega_{n} \omega\right\rangle\right\}$ (resp. $\langle\llbracket p, B \rrbracket, \gamma \omega\rangle)$.

A run $\rho$ of a SABPDS $\mathcal{B P}$ starting form an initial configuration $\left\langle\llbracket p_{0}, B_{0} \rrbracket, \omega_{0}\right\rangle$ is a tree whose root is labelled by $\left\langle\llbracket p_{0}, B_{0} \rrbracket, \omega_{0}\right\rangle$, and whose other nodes are labelled by elements in $P \times \mathcal{B} \times \Gamma^{*}$. If a node of $\rho$ is labelled by a configuration $\langle\llbracket p, B \rrbracket, \omega\rangle$ and has $n$ children labelled by $\left\langle\llbracket p_{1}, B_{1} \rrbracket, \omega_{1}\right\rangle, \ldots,\left\langle\llbracket p_{n}, B_{n} \rrbracket, \omega_{n}\right\rangle$ respectively, then, $\langle\llbracket p, B \rrbracket, \omega\rangle$ must be a predecessor of $\left\{\left\langle\llbracket p_{1}, B_{1} \rrbracket, \omega_{1}\right\rangle, \ldots,\left\langle\llbracket p_{n}, B_{n} \rrbracket, \omega_{n}\right\rangle\right\}$ in $\mathcal{B P}$. A path of a run $\rho$ is an infinite sequence of configurations $c_{0} c_{1} c_{2} \ldots$ s.t. $c_{0}$ is the root of $\rho$ and $c_{i+1}$ is one of the children of $c_{i}$ for every $i \geq 0$. A path is accepting iff it visits infinitely often configurations with control locations in $F$. A run $\rho$ is accepting iff every path of $\rho$ is accepting. The language of $\mathcal{B P}$, $\mathcal{L}(\mathcal{B P})$, is the set of configurations $c$ s.t. $\mathcal{B P}$ has an accepting run starting from c.

$\mathcal{B P}$ defines the reachability relation $\Rightarrow_{\mathcal{B P}}: 2^{(P \times \mathcal{B}) \times \Gamma^{*}} \rightarrow 2^{(P \times \mathcal{B}) \times \Gamma^{*}}$ as follows: (1) $c \Rightarrow_{\mathcal{B P}}\{c\}$ for every $c \in P \times \mathcal{B} \times \Gamma^{*},(2) c \Rightarrow_{\mathcal{B} \mathcal{P}} C$ if $C$ is an immediate 
successor of $c$; (3) if $c \Rightarrow_{\mathcal{B P}}\left\{c_{1}, c_{2}, \ldots, c_{n}\right\}$ and $c_{i} \Rightarrow_{\mathcal{B P}} C_{i}$ for every $1 \leq i \leq n$, then $c \Rightarrow{ }_{\mathcal{B P}} \bigcup_{i=1}^{n} C_{i}$. Given $c_{0} \Rightarrow{ }_{\mathcal{B P}} C^{\prime}$, then, $\mathcal{B P}$ has an accepting run from $c_{0}$ iff $\mathcal{B P}$ has an accepting run from $c^{\prime}$ for every $c^{\prime} \in C^{\prime}$.

Theorem 3. [10] The membership problem of SABPDS can be solved effectively.

Functions of $\mathbb{R}$. In what follows, we define several functions of $\mathbb{R}$ which will be used in the next sections. These functions were first defined in [10].

1. $i d(B)=\{B\}$. This is the identity function.

2 .

$$
\begin{aligned}
& \operatorname{equal}\left(B_{1}, \ldots, B_{n}\right)= \\
& \begin{cases}\left\{B_{1}\right\} & \text { if } B_{i}=B_{j} \text { for every } 1 \leq i, j \leq n ; \\
\emptyset & \text { otherwise }\end{cases}
\end{aligned}
$$

This function checks whether all the environments are equal and returns $\left\{B_{1}\right\}$ (which is also equal to $B_{i}$ for every $i$ ). Otherwise, it returns the emptyset.

3.

$$
\begin{aligned}
& \text { meet }_{\left\{c_{1}, \ldots, c_{n}\right\}}^{x}\left(B_{1}, \ldots, B_{n}\right)= \\
& \begin{cases}A b s_{x}\left(B_{1}\right) & \text { if } B_{i}(x)=c_{i} \text { for } 1 \leq i \leq n, \\
\emptyset & \text { and } B_{i}(y)=B_{j}(y) \text { for } y \neq x, 1 \leq i, j \leq n\end{cases}
\end{aligned}
$$

This function checks whether (1) $B_{i}(x)=c_{i}$ for every $1 \leq i \leq n$ (2) for every $y \neq x$; every $1 \leq i, j \leq n B_{i}(y)=B_{j}(y)$. If the conditions are satisfied, it returns $A b s_{x}\left(B_{1}\right)^{1}$, otherwise it returns the emptyset.

4 .

$$
\operatorname{join}_{c}^{x}\left(B_{1}, \ldots, B_{n}\right)= \begin{cases}B_{1} & \text { if } B_{i}(x)=c \text { for } 1 \leq i \leq n \\ & \text { and } B_{i}=B_{j} \text { for } 1 \leq i, j \leq n \\ \emptyset & \text { otherwise }\end{cases}
$$

This function checks whether $B_{i}(x)=c$ for every $i$. If this condition is satisfied, equal $\left(B_{1}, \ldots, B_{n}\right)$ is returned, otherwise, the emptyset is returned.

5 .

$$
j o i \neg_{c}^{\neg x}\left(B_{1}, \ldots, B_{n}\right)= \begin{cases}B_{1} & \text { if } B_{i}(x) \neq c \text { for } 1 \leq i \leq n \\ & \text { and } B_{i}=B_{j} \text { for } 1 \leq i, j \leq n ; \\ \emptyset & \text { otherwise }\end{cases}
$$

This function checks whether $B_{i}(x) \neq c$ for every $i$. If this condition is satisfied, equal $\left(B_{1}, \ldots, B_{n}\right)$ is returned, otherwise, the emptyset is returned.

${ }^{1} A b s_{x}\left(B_{1}\right)$ is as defined in Section 3.1 


\subsection{From SBPCARET model checking of PDSs to the membership problem in SABPDSs}

Let $\mathcal{P}=(P, \Gamma, \Delta)$ be a PDS. We suppose w.l.o.g. that $\mathcal{P}$ has a bottom stack symbol $\sharp$ that is never popped from the stack. Let AP be a set of atomic propositions. Let $\varphi$ be a SBPCARET formula over $A P, \lambda: P \longrightarrow 2^{A P_{\mathcal{D}}}$ be a labelling function. Given a configuration $\left\langle p_{0}, \omega_{0}\right\rangle$, we propose in this section an algorithm to check whether $\left\langle p_{0}, \omega_{0}\right\rangle \vDash_{\lambda} \varphi$, i.e., whether there exists an environment $B$ s.t. $\left\langle p_{0}, \omega_{0}\right\rangle \vDash_{\lambda}^{B} \varphi$. Intuitively, we compute an $\operatorname{SABPDS} \mathcal{B P} \varphi$ s.t. $\langle p, \omega\rangle \vDash_{\lambda}^{B} \varphi$ iff $\langle\llbracket(p, \varphi), B \rrbracket, \omega\rangle \in \mathcal{L}(\mathcal{B P} \varphi)$ for every $p \in P, \omega \in \Gamma^{*}, B \in \mathcal{B}$. Then, to check if $\left\langle p_{0}, \omega_{0}\right\rangle \vDash_{\lambda} \varphi$, we will check whether there exists a $B \in \mathcal{B}$ s.t. $\left\langle\llbracket\left(p_{0}, \varphi\right), B \rrbracket, \omega_{0}\right\rangle \in \mathcal{L}(\mathcal{B P} \varphi)$.

Let $\operatorname{Reg}^{+}(\varphi)=\left\{e_{1}, \ldots, e_{k}\right\}$ and $\operatorname{Reg}^{-}(\varphi)=\left\{e_{k+1}, \ldots, e_{m}\right\}$. Using Theorems 1 and 2 ; for every $1 \leq i \leq k$, we can compute a VA $M_{e_{i}}=\left(Q_{e_{i}}, \Gamma, \delta_{e_{i}}, s_{e_{i}}, F_{e_{i}}\right)$ s.t. $L\left(M_{e_{i}}\right)=L\left(e_{i}\right)$. In addition, for every $k+1 \leq j \leq m$, we can compute a VA $M_{\neg e_{j}}=\left(Q_{\neg e_{j}}, \Gamma, \delta_{\neg e_{j}}, s_{\neg e_{j}}, F_{\neg e_{j}}\right)$ s.t. $L\left(M_{\neg e_{j}}\right)=\left(P \times \Gamma^{*}\right) \times \mathcal{B} \backslash L\left(e_{j}\right)$. Let $\mathcal{M}$ be the union of all these automata, $\mathcal{S}$ and $\mathcal{F}$ be respectively the union of all states and final states of these automata.

Let $\mathcal{B P} \varphi=\left(P^{\prime}, \Gamma^{\prime}, \Delta^{\prime}, F\right)$ be the SABPDS defined as follows:

- $P^{\prime}=P \cup(P \times C l(\varphi)) \cup \mathcal{S} \cup\left\{p_{\perp}\right\}$

- $\Gamma^{\prime}=\Gamma \cup(\Gamma \times C l(\varphi)) \cup\left\{\gamma_{\perp}\right\}$

- $F=F_{1} \cup F_{2} \cup F_{3} \cup F_{4}$ where

- $F_{1}=\left\{\llbracket\left(p, b\left(\alpha_{1}, \ldots, \alpha_{n}\right)\right), \beta \rrbracket \mid b\left(\alpha_{1}, \ldots, \alpha_{n}\right) \in A P^{+}(\varphi)\right.$, and $\beta=\{B \in$ $\left.\mathcal{B} \mid b\left(B\left(\alpha_{1}\right), \ldots, B\left(\alpha_{n}\right)\right) \in \lambda(p)\right\}$

- $F_{2}=\left\{\llbracket\left(p, \neg b\left(\alpha_{1}, \ldots, \alpha_{n}\right)\right), \beta \rrbracket \mid b\left(\alpha_{1}, \ldots, \alpha_{n}\right) \in A P^{-}(\varphi)\right.$, and $\beta=\{B \in$ $\left.\mathcal{B} \mid b\left(B\left(\alpha_{1}\right), \ldots, B\left(\alpha_{n}\right)\right) \notin \lambda(p)\right\}$

- $F_{3}=P \times C l_{R}(\varphi) \times \mathcal{B}$ where $C l_{R}(\varphi)$ is the set of formulas of $C l(\varphi)$ in the form $E\left[\varphi_{1} R^{v} \varphi_{2}\right]$ or $A\left[\varphi_{1} R^{v} \varphi_{2}\right](v \in\{g, a\})$

- $F_{4}=\mathcal{F} \times \mathcal{B}$

The transition relation $\Delta^{\prime}$ is the smallest set of transition rules defined as follows: For every $p \in P, \phi \in C l(\varphi), \gamma \in \Gamma$ and $t \in\{$ call, ret, int $\}$ :

(ћ1) If $\phi=b\left(\alpha_{1}, \ldots, \alpha_{n}\right)$, then, $\left.\left.\left.\langle(p, \phi\rangle), \gamma\right\rangle \stackrel{i d}{\hookrightarrow}\langle\mid p, \phi\rangle\right), \gamma\right\rangle \in \Delta^{\prime}$

(ћ2) If $\phi=\neg b\left(\alpha_{1}, \ldots, \alpha_{n}\right)$, then, $\left.\left.\langle(p, \phi\rangle), \gamma\right\rangle \stackrel{i d}{\longrightarrow}\langle(p, \phi\rangle), \gamma\right\rangle \in \Delta^{\prime}$

(ћ3) If $\phi=\phi_{1} \wedge \phi_{2}$, then, $\left.\langle(p, \phi\rangle), \gamma\right\rangle \stackrel{\text { equal }}{\longrightarrow}\left[\left\langle\left(\mid p, \phi_{1}\right), \gamma\right\rangle,\left\langle\left(p, \phi_{2}\right\rangle, \gamma\right\rangle\right] \in \Delta^{\prime}$

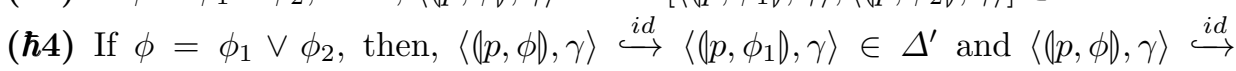
$\left\langle\left(p, \phi_{2}\right), \gamma\right\rangle \in \Delta^{\prime}$

(ћ5) If $\phi=\exists x \phi_{1}$, then, $\langle(p, \phi), \gamma\rangle \stackrel{\text { meet }_{\{c\}}^{x}}{\longrightarrow}\left\langle\left(p, \phi_{1}|, \gamma\rangle \in \Delta^{\prime}\right.\right.$ for every $c \in \mathcal{D}$

(ћ6) If $\phi=\forall x \phi_{1}$, then, $\langle(\mid p, \phi), \gamma\rangle \stackrel{\text { meet }_{\mathcal{D}}^{x}}{\longrightarrow}\left[\left\langle\left(\mid p, \phi_{1}\right), \gamma\right\rangle, \ldots,\left\langle\left(\mid p, \phi_{1}\right), \gamma\right\rangle\right] \in \Delta^{\prime}$ where $\left\langle\left\langle p, \phi_{1}\right\rangle, \gamma\right\rangle$ is repeated $m$ times in the right-hand side, where $m$ is the number of elements in $\mathcal{D}$

(ћ7) If $\phi=E X^{g} \phi_{1}$, then

$\langle(p, \phi\rangle) \gamma\rangle \stackrel{i d}{\hookrightarrow}\left\langle\left(\left|q, \phi_{1} D, \omega\right\rangle \in \Delta^{\prime}\right.\right.$ for every $\langle p, \gamma\rangle$

$\stackrel{t}{\rightarrow}\langle q, \omega\rangle \in \Delta$ 
( $\hbar 8)$ If $\phi=A X^{g} \phi_{1}$, then,

$\langle(p, \phi), \gamma\rangle \stackrel{\text { equal }}{\longrightarrow}\left[\left\langle\left(q_{1}, \phi_{1}\right\rangle, \omega_{1}\right\rangle, \ldots,\left\langle\left(\left|q_{n}, \phi_{1}\right\rangle, \omega_{n}\right\rangle\right] \in \Delta^{\prime}\right.$, where for every $1 \leq$ $i \leq n,\langle p, \gamma\rangle \stackrel{t}{\rightarrow}\left\langle q_{i}, \omega_{i}\right\rangle \in \Delta$ and these transitions are all the transitions of $\Delta$ that are in the form $\langle p, \gamma\rangle \stackrel{t}{\rightarrow}\langle q, \omega\rangle$ that have $\langle p, \gamma\rangle$ on the left hand side.

(ћ9) If $\phi=E X^{a} \phi_{1}$, then,
(a) $\langle(p, \phi), \gamma\rangle \stackrel{\text { id }}{\longrightarrow}\left\langle q, \gamma^{\prime}\left(\gamma^{\prime \prime}, \phi_{1}\right)\right\rangle \in \Delta^{\prime}$ for every $\langle p, \gamma\rangle \stackrel{\text { call }}{\longrightarrow}\left\langle q, \gamma^{\prime} \gamma^{\prime \prime}\right\rangle \in \Delta$
(b) $\langle(p, \phi\rangle, \gamma\rangle \stackrel{\text { id }}{\hookrightarrow}\left\langle\mid q q, \phi_{1} D, \omega\right\rangle \in \Delta^{\prime}$ for every $\langle p, \gamma\rangle \stackrel{\text { int }}{\longrightarrow}\langle q, \omega\rangle \in \Delta$
(c) $\langle(p, \phi), \gamma\rangle \stackrel{i d}{\hookrightarrow}\left\langle p_{\perp}, \gamma_{\perp}\right\rangle \in \Delta^{\prime}$ for every $\langle p, \gamma\rangle \stackrel{\text { ret }}{\longrightarrow}\left\langle q^{\prime}, \epsilon\right\rangle \in \Delta$

(ћ10) If $\phi=A X^{a} \phi_{1}$, then,

$\langle(p, \phi\rangle), \gamma\rangle \stackrel{\text { equal }}{\longrightarrow}\left[\left\langle p_{1}, \gamma_{1}^{\prime}\right|\left\langle\gamma_{1}^{\prime \prime}, \phi_{1} D\right\rangle, \ldots,\left\langle p_{m}, \gamma_{m}^{\prime}|| \gamma_{m}^{\prime \prime}, \phi_{1} D\right\rangle,\left\langle\left(\left|q_{1}, \phi_{1} D, \omega_{1}\right\rangle, \ldots,\left\langle\left(\left|q_{n}, \phi_{1} D, \omega_{n}\right\rangle\right.\right.\right.\right.\right.$, $\left.\left\langle p_{\perp}, \gamma_{\perp}\right\rangle, \ldots,\left\langle p_{\perp}, \gamma_{\perp}\right\rangle\right] \in \Delta^{\prime}$, where $\left\langle p_{\perp}, \gamma_{\perp}\right\rangle$ is repeated $k$ times in the righthand side s.t.:

(a) for every $1 \leq i \leq m,\langle p, \gamma\rangle \stackrel{\text { call }}{\longrightarrow}\left\langle p_{i}, \gamma_{i}^{\prime} \gamma_{i}^{\prime \prime}\right\rangle \in \Delta$ and these transitions are all the transitions of $\Delta$ that are in the form $\langle p, \gamma\rangle \stackrel{\text { call }}{\longrightarrow}\left\langle q, \gamma^{\prime} \gamma^{\prime \prime}\right\rangle$ that have $\langle p, \gamma\rangle$ on the left hand side.

(b) for every $1 \leq i \leq n,\langle p, \gamma\rangle \stackrel{\text { int }}{\longrightarrow}\left\langle q_{i}, \omega_{i}\right\rangle \in \Delta$ and these transitions are all the transitions of $\Delta$ that are in the form $\langle p, \gamma\rangle \stackrel{\text { int }}{\longrightarrow}\langle q, \omega\rangle$ that have $\langle p, \gamma\rangle$ on the left hand side.

(c) for every $1 \leq i \leq k,\langle p, \gamma\rangle \stackrel{\text { ret }}{\longrightarrow}\left\langle q_{i}^{\prime}, \epsilon\right\rangle \in \Delta$ and these transitions are all the transitions of $\Delta$ that are in the form $\langle p, \gamma\rangle \stackrel{\text { ret }}{\longrightarrow}\left\langle q^{\prime}, \epsilon\right\rangle$ that have $\langle p, \gamma\rangle$ on the left hand side.

(ћ11) If $\phi=E\left[\phi_{1} U^{g} \phi_{2}\right]$, then,

(a) $\langle(p, \phi), \gamma\rangle \stackrel{i d}{\hookrightarrow}\left\langle\left(p, \phi_{2}\right), \gamma\right\rangle \in \Delta^{\prime}$

(b) $\langle(p, \phi\rangle), \gamma\rangle \stackrel{\text { equal }}{\longrightarrow}\left[\left\langle\left(p, \phi_{1}\right\rangle, \gamma\right\rangle,\langle(|q, \phi\rangle), \omega\rangle\right] \in \Delta^{\prime}$ for every $\langle p, \gamma\rangle \stackrel{t}{\rightarrow}\langle q, \omega\rangle \in$ $\Delta$

( $\hbar 12)$ If $\phi=E\left[\phi_{1} U^{a} \phi_{2}\right]$, then,

(a) $\langle(p, \phi), \gamma\rangle \stackrel{i d}{\hookrightarrow}\left\langle\left(p, \phi_{2}\right), \gamma\right\rangle \in \Delta^{\prime}$

(b) $\langle\mid p, \phi\rangle, \gamma\rangle \stackrel{\text { equal }}{\longrightarrow}\left[\left\langle\mid p, \phi_{1} D, \gamma\right\rangle,\left\langle q, \gamma^{\prime} \mid \gamma^{\prime \prime}, \phi D\right\rangle\right] \in \Delta^{\prime}$ for every $\langle p, \gamma\rangle \stackrel{\text { call }}{\longrightarrow}$ $\left\langle q, \gamma^{\prime} \gamma^{\prime \prime}\right\rangle \in \Delta$

(c) $\langle(p, \phi\rangle), \gamma\rangle \stackrel{\text { equal }}{\longrightarrow}\left[\left\langle\left(p, \phi_{1} D, \gamma\right\rangle,\langle(|q, \phi\rangle, \omega\rangle] \in \Delta^{\prime}\right.\right.$ for every $\langle p, \gamma\rangle \stackrel{\text { int }}{\longrightarrow}\langle q, \omega\rangle \in$ $\Delta$

(d) $\langle(p, \phi), \gamma\rangle \stackrel{i d}{\longrightarrow}\left\langle p_{\perp}, \gamma_{\perp}\right\rangle \in \Delta^{\prime}$ for every $\langle p, \gamma\rangle \stackrel{\text { ret }}{\longrightarrow}\left\langle q^{\prime}, \epsilon\right\rangle \in \Delta$

(ఓ13) If $\phi=A\left[\phi_{1} U^{g} \phi_{2}\right]$, then,

(a) $\langle(p, \phi), \gamma\rangle \stackrel{i d}{\longrightarrow}\left\langle\left(p, \phi_{2}\right), \gamma\right\rangle \in \Delta^{\prime}$

(b) $\langle(p, \phi\rangle), \gamma\rangle \stackrel{\text { equal }}{\longrightarrow}\left[\left\langle\left(p, \phi_{1}\right\rangle, \gamma\right\rangle ;\left\langle\left(\left|q_{1}, \phi\right\rangle, \omega_{1}\right\rangle, \ldots,\left\langle\left(\left|q_{n}, \phi\right\rangle, \omega_{n}\right\rangle\right] \in \Delta^{\prime}\right.\right.$ where for every $1 \leq i \leq n,\langle p, \gamma\rangle \stackrel{t}{\rightarrow}\left\langle q_{i}, \omega_{i}\right\rangle \in \Delta$ and these transitions are all the transitions of $\Delta$ that are in the form $\langle p, \gamma\rangle \stackrel{t}{\rightarrow}\langle q, \omega\rangle$ that have $\langle p, \gamma\rangle$ on the left hand side.

(ћ14) If $\phi=A\left[\phi_{1} U^{a} \phi_{2}\right]$, then,

(a) $\langle(p, \phi), \gamma\rangle \stackrel{i d}{\hookrightarrow}\left\langle\left(p, \phi_{2}\right), \gamma\right\rangle \in \Delta^{\prime}$ 
(b) $\left.\langle(p, \phi), \gamma\rangle \stackrel{\text { equal }}{\longrightarrow}\left[\left\langle\left(\mid p, \phi_{1}\right), \gamma\right\rangle ;\left\langle p_{1}, \gamma_{1}^{\prime}\right| \gamma_{1}^{\prime \prime}, \phi\right)\right\rangle, \ldots,\left\langle p_{m}, \gamma_{m}^{\prime} \mid \gamma_{m}^{\prime \prime}, \phi 0\right\rangle ;\left\langle\left(\left|q_{1}, \phi 0, \omega_{1}\right\rangle, \ldots\right.\right.$ $\left.\left\langle\left(q_{n}, \phi\right), \omega_{n}\right\rangle,\left\langle p_{\perp}, \gamma_{\perp}\right\rangle, \ldots,\left\langle p_{\perp}, \gamma_{\perp}\right\rangle\right] \in \Delta^{\prime}$, where $\left\langle p_{\perp}, \gamma_{\perp}\right\rangle$ is repeated $k$ times in the right-hand side s.t.:

- for every $1 \leq i \leq m,\langle p, \gamma\rangle \stackrel{\text { call }}{\longrightarrow}\left\langle p_{i}, \gamma_{i}^{\prime} \gamma_{i}^{\prime \prime}\right\rangle \in \Delta$ and these transitions are all the transitions of $\Delta$ that are in the form $\langle p, \gamma\rangle \stackrel{\text { call }}{\longrightarrow}\left\langle q, \gamma^{\prime} \gamma^{\prime \prime}\right\rangle$ that have $\langle p, \gamma\rangle$ on the left hand side.

- for every $1 \leq i \leq n,\langle p, \gamma\rangle \stackrel{i n t}{\longrightarrow}\left\langle q_{i}, \omega_{i}\right\rangle \in \Delta$ and these transitions are all the transitions of $\Delta$ that are in the form $\langle p, \gamma\rangle \stackrel{\text { int }}{\longrightarrow}\langle q, \omega\rangle$ that have $\langle p, \gamma\rangle$ on the left hand side.

- for every $1 \leq i \leq k,\langle p, \gamma\rangle \stackrel{\text { ret }}{\longrightarrow}\left\langle q_{i}^{\prime}, \epsilon\right\rangle \in \Delta$ and these transitions are all the transitions of $\Delta$ that are in the form $\langle p, \gamma\rangle \stackrel{\text { ret }}{\longrightarrow}\left\langle q^{\prime}, \epsilon\right\rangle$ that have $\langle p, \gamma\rangle$ on the left hand side.

(ћ15) If $\phi=E\left[\phi_{1} R^{g} \phi_{2}\right]$, then, we add to $\Delta^{\prime}$ the rule:

(a) $\langle(p, \phi), \gamma\rangle \stackrel{\text { equal }}{\longrightarrow}\left[\left\langle\left(\mid p, \phi_{2}\right), \gamma\right\rangle,\left\langle\left(\mid p, \phi_{1}\right), \gamma\right\rangle\right] \in \Delta^{\prime}$

(b) $\langle(p, \phi), \gamma\rangle \stackrel{\text { equal }}{\longrightarrow}\left[\left\langle\left(\mid p, \phi_{2}\right), \gamma\right\rangle,\langle(\mid q, \phi), \omega\rangle\right] \in \Delta^{\prime}$ for every $\langle p, \gamma\rangle \stackrel{t}{\rightarrow}\langle q, \omega\rangle \in$ $\Delta$

(ћ16) If $\phi=A\left[\phi_{1} R^{g} \phi_{2}\right]$, then, we add to $\Delta^{\prime}$ the rule:

(a) $\langle(p, \phi), \gamma\rangle \stackrel{\text { equal }}{\longrightarrow}\left[\left\langle\left(\mid p, \phi_{2}\right), \gamma\right\rangle,\left\langle\left(\mid p, \phi_{1}\right), \gamma\right\rangle\right] \in \Delta^{\prime}$

(b) $\langle(p, \phi), \gamma\rangle \stackrel{\text { equal }}{\longrightarrow}\left[\left\langle\left(\mid p, \phi_{2}\right), \gamma\right\rangle ;\left\langle\left(\mid q_{1}, \phi\right), \omega_{1}\right\rangle, \ldots,\left\langle\left(\mid q_{n}, \phi\right), \omega_{n}\right\rangle\right] \in \Delta^{\prime}$ where for every $1 \leq i \leq n,\langle p, \gamma\rangle \stackrel{t}{\rightarrow}\left\langle q_{i}, \omega_{i}\right\rangle \in \Delta$ and these transitions are all the transitions of $\Delta$ that are in the form $\langle p, \gamma\rangle \stackrel{t}{\rightarrow}\langle q, \omega\rangle$ that have $\langle p, \gamma\rangle$ on the left hand side.

(ћ17) If $\phi=E\left[\phi_{1} R^{a} \phi_{2}\right]$, then,

(a) $\langle(\mid p, \phi), \gamma\rangle \stackrel{\text { equal }}{\longrightarrow}\left[\left\langle\left(\mid p, \phi_{2}\right), \gamma\right\rangle,\left\langle\left(\mid p, \phi_{1}\right), \gamma\right\rangle\right] \in \Delta^{\prime}$

(b) $\langle(p, \phi), \gamma\rangle \stackrel{\text { equal }}{\longrightarrow}\left[\left\langle\left(p, \phi_{2}\right), \gamma\right\rangle,\left\langle q, \gamma^{\prime}\left(\gamma^{\prime \prime}, \phi\right)\right\rangle\right] \in \Delta^{\prime}$ for every $\langle p, \gamma\rangle \stackrel{\text { call }}{\longrightarrow}$ $\left\langle q, \gamma^{\prime} \gamma^{\prime \prime}\right\rangle \in \Delta$

(c) $\langle(p, \phi), \gamma\rangle \stackrel{\text { equal }}{\longrightarrow}\left[\left\langle\left(\mid p, \phi_{2}\right), \gamma\right\rangle,\langle(\mid q, \phi), \omega\rangle\right] \in \Delta^{\prime}$ for every $\langle p, \gamma\rangle \stackrel{\text { int }}{\longrightarrow}\langle q, \omega\rangle \in$ $\Delta$

(d) $\langle(p, \phi), \gamma\rangle \stackrel{i d}{\hookrightarrow}\left\langle p_{\perp}, \gamma_{\perp}\right\rangle \in \Delta^{\prime}$ for every $\langle p, \gamma\rangle \stackrel{\text { ret }}{\longrightarrow}\left\langle q^{\prime}, \epsilon\right\rangle \in \Delta$

(ћ18) If $\phi=A\left[\phi_{1} R^{a} \phi_{2}\right]$, then,

(a) $\langle(p p, \phi), \gamma\rangle \stackrel{\text { equal }}{\longrightarrow}\left[\left\langle\left(\mid p, \phi_{2}\right), \gamma\right\rangle,\left\langle\left(\mid p, \phi_{1}\right), \gamma\right\rangle\right] \in \Delta^{\prime}$

(b) $\langle(p p, \phi), \gamma\rangle \stackrel{\text { equal }}{\longrightarrow}\left[\left\langle\left(\mid p, \phi_{2}\right), \gamma\right\rangle ;\left\langle p_{1}, \gamma_{1}^{\prime} \mid \gamma_{1}^{\prime \prime}, \phi 0\right\rangle, \ldots,\left\langle p_{m}, \gamma_{m}^{\prime}\left(\gamma_{m}^{\prime \prime}, \phi\right)\right\rangle ;\left\langle\left(\mid q_{1}, \phi\right), \omega_{1}\right\rangle\right.$ $\left., \ldots,\left\langle\left(\mid q_{n}, \phi\right), \omega_{n}\right\rangle,\left\langle p_{\perp}, \gamma_{\perp}\right\rangle, \ldots,\left\langle p_{\perp}, \gamma_{\perp}\right\rangle\right] \in \Delta^{\prime}$, where $\left\langle p_{\perp}, \gamma_{\perp}\right\rangle$ is repeated $k$ times in the right-hand side s.t.:

- for every $1 \leq i \leq m,\langle p, \gamma\rangle \stackrel{\text { call }}{\longrightarrow}\left\langle p_{i}, \gamma_{i}^{\prime} \gamma_{i}^{\prime \prime}\right\rangle \in \Delta$ and these transitions are all the transitions of $\Delta$ that are in the form $\langle p, \gamma\rangle \stackrel{\text { call }}{\longrightarrow}\left\langle q, \gamma^{\prime} \gamma^{\prime \prime}\right\rangle$ that have $\langle p, \gamma\rangle$ on the left hand side.

- for every $1 \leq i \leq n,\langle p, \gamma\rangle \stackrel{\text { int }}{\longrightarrow}\left\langle q_{i}, \omega_{i}\right\rangle \in \Delta$ and these transitions are all the transitions of $\Delta$ that are in the form $\langle p, \gamma\rangle \stackrel{\text { int }}{\longrightarrow}\langle q, \omega\rangle$ that have $\langle p, \gamma\rangle$ on the left hand side. 
- for every $1 \leq i \leq k,\langle p, \gamma\rangle \stackrel{\text { ret }}{\longrightarrow}\left\langle q_{i}^{\prime}, \epsilon\right\rangle \in \Delta$ and these transitions are all the transitions of $\Delta$ that are in the form $\langle p, \gamma\rangle \stackrel{r e t}{\longrightarrow}\left\langle q^{\prime}, \epsilon\right\rangle$ that have $\langle p, \gamma\rangle$ on the left hand side.

(ћ19) for every $\langle p, \gamma\rangle \stackrel{\text { ret }}{\longrightarrow}\langle q, \epsilon\rangle \in \Delta$ :

- $\left.\left\langle q, \mid \gamma^{\prime \prime}, \phi_{1}\right\rangle\right\rangle \stackrel{i d}{\longrightarrow}\left\langle\left(\mid q, \phi_{1}\right), \gamma^{\prime \prime}\right\rangle \in \Delta^{\prime}$ for every $\gamma^{\prime \prime} \in \Gamma, \phi_{1} \in C l(\varphi)$

(市20) $\left\langle p_{\perp}, \gamma_{\perp}\right\rangle \stackrel{i d}{\hookrightarrow}\left\langle p_{\perp}, \gamma_{\perp}\right\rangle \in \Delta^{\prime}$

(ћ21) for every $\langle p, \gamma\rangle \stackrel{t}{\rightarrow}\langle q, \omega\rangle \in \Delta:\langle p, \gamma\rangle \stackrel{i d}{\rightarrow}\langle q, \omega\rangle \in \Delta^{\prime}$

(\$22) If $\phi=e, e$ is a regular expression, then, $\langle(p, \phi), \gamma\rangle \stackrel{i d}{\hookrightarrow}\left\langle s_{e}, \gamma\right\rangle \in \Delta^{\prime}$

(ћ23) If $\phi=\neg e, e$ is a regular expression, then, $\langle(p, \phi), \gamma\rangle \stackrel{i d}{\hookrightarrow}\left\langle s_{\neg e}, \gamma\right\rangle \in \Delta^{\prime}$

(ћ24) for every transition $q \stackrel{\alpha}{\rightarrow}\left\{q_{1}, . ., q_{n}\right\}$ in $\mathcal{M}:\langle q, \gamma\rangle \stackrel{\mathbb{R}}{\hookrightarrow}\left[\left\langle q_{1}, \epsilon\right\rangle, \ldots,\left\langle q_{n}, \epsilon\right\rangle\right] \in \Delta^{\prime}$, where:

(a) $\mathbb{R}=$ equal iff $\alpha=\gamma$

(b) $\mathbb{R}=j_{\text {oin }}^{x}$ iff $\alpha=x \in \mathcal{X}$

(c) $\left.\mathbb{R}=\operatorname{join}_{\gamma}\right\urcorner^{x}$ iff $\alpha=\neg x$ and $x \in \mathcal{X}$

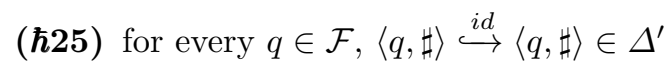

Roughly speaking, the SABPDS $\mathcal{B P}_{\varphi}$ is a kind of product between $\mathcal{P}$ and the SBPCARET formula $\varphi$ which ensures that $\mathcal{B P}_{\varphi}$ has an accepting run from $\langle\llbracket(p, \varphi\rangle, B \rrbracket, \omega\rangle$ iff the configuration $\langle p, \omega\rangle$ satisfies $\varphi$ under the environment $B$. The form of the control locations of $\mathcal{B P} \mathcal{P}_{\varphi}$ is $\llbracket(p, \phi), B \rrbracket$ where $\phi \in C l(\varphi), B \in \mathcal{B}$. Let us explain the intuition behind our construction:

- If $\phi=b\left(\alpha_{1}, \ldots, \alpha_{n}\right)$, then, for every $\omega \in \Gamma^{*},\langle p, \omega\rangle \models_{\lambda}^{B} \phi$ iff $b\left(B\left(\alpha_{1}\right), \ldots, B\left(\alpha_{n}\right)\right) \in$ $\lambda(p)$. Thus, for such a $B, \mathcal{B P} \varphi$ should have an accepting run from $\left\langle\llbracket\left(p, b\left(\alpha_{1}, \ldots, \alpha_{n}\right)\right), B \rrbracket, \omega\right\rangle$ iff $b\left(B\left(\alpha_{1}\right), \ldots, B\left(\alpha_{n}\right)\right) \in \lambda(p)$. This is ensured by the transition rules in $(\hbar 1)$ which add a loop at $\left\langle\llbracket\left(p, b\left(\alpha_{1}, \ldots, \alpha_{n}\right) D, B \rrbracket, \omega\right\rangle\right.$ and the fact that $\llbracket\left(p, b\left(\alpha_{1}, \ldots, \alpha_{n}\right)\right), B \rrbracket \in F$ (because it is in $\left.F_{1}\right)$. The function $i d$ in $(\hbar 1)$ ensures that the environments before and after are the same.

- If $\phi=\neg b\left(\alpha_{1}, \ldots, \alpha_{n}\right)$, then, for every $\omega \in \Gamma^{*},\langle p, \omega\rangle \models_{\lambda}^{B} \phi$ iff $b\left(B\left(\alpha_{1}\right), \ldots, B\left(\alpha_{n}\right)\right) \notin$ $\lambda(p)$. Thus, for such a $B, \mathcal{B P} \varphi$ should have an accepting run from $\left\langle\llbracket\left(p, \neg b\left(\alpha_{1}, \ldots, \alpha_{n}\right) D, B \rrbracket, \omega\right\rangle\right.$ iff $b\left(B\left(\alpha_{1}\right), \ldots, B\left(\alpha_{n}\right)\right) \notin \lambda(p)$. This is ensured by the transition rules in $(\hbar 2)$ which add a loop at $\left\langle\llbracket\left(p, \neg b\left(\alpha_{1}, \ldots, \alpha_{n}\right)\right\rangle, B \rrbracket, \omega\right\rangle$ and the fact that $\llbracket\left(p, \neg b\left(\alpha_{1}, \ldots, \alpha_{n}\right)\right), B \rrbracket \in F$ (because it is in $\left.F_{2}\right)$. The function $i d$ in ( $(\boldsymbol{\pi} 2)$ ensures that the environments before and after are the same.

- If $\phi=\phi_{1} \wedge \phi_{2}$, then, for every $\omega \in \Gamma^{*},\langle p, \omega\rangle \vDash_{\lambda}^{B} \phi$ iff $\left(\langle p, \omega\rangle \vDash_{\lambda}^{B} \phi_{1}\right.$ and $\left.\langle p, \omega\rangle \vDash_{\lambda}^{B} \phi_{2}\right)$. This is ensured by the transition rules in ( $\left.\hbar 3\right)$ stating that $\mathcal{B} \mathcal{P}_{\varphi}$ has an accepting run from $\left\langle\llbracket\left(\mid p, \phi_{1} \wedge \phi_{2}\right), B \rrbracket, \omega\right\rangle$ iff $\mathcal{B P} \mathcal{P}_{\varphi}$ has an accepting

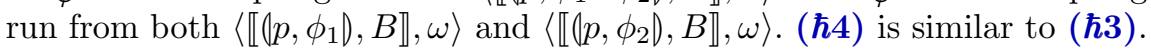

- If $\phi=\exists x \phi_{1}$, then, for every $\omega \in \Gamma^{*},\langle p, \omega\rangle \vDash_{\lambda}^{B} \phi$ iff there exists $c \in \mathcal{D}$ s.t. $\langle p, \omega\rangle \vDash_{\lambda}^{B[x \leftarrow c]} \phi_{1}$. This is ensured by the transition rules in ( $\left.\hbar 5\right)$ stating that $\mathcal{B P} \mathcal{P}_{\varphi}$ has an accepting run from $\left\langle\llbracket\left(p, \exists x \phi_{1}\right), B \rrbracket, \omega\right\rangle$ iff there exists $c \in$ $\mathcal{D}$ s.t. $\mathcal{B P} \varphi$ has an accepting run from $\left\langle\llbracket \mid\left(p, \phi_{1}\right), B[x \leftarrow c] \rrbracket, \omega\right\rangle$ since $B \in$ $\operatorname{meet}_{\{c\}}^{x}(B[x \leftarrow c])$ 
- If $\phi=\forall x \phi_{1}$, then, for every $\omega \in \Gamma^{*},\langle p, \omega\rangle \vDash_{\lambda}^{B} \phi$ iff for every $c \in \mathcal{D}$, $\langle p, \omega\rangle \vDash_{\lambda}^{B[x \leftarrow c]} \phi_{1}$. This is ensured by the transition rules in ( $\left.\hbar 6\right)$ stating that $\mathcal{B P}_{\varphi}$ has an accepting run from $\left\langle\llbracket\left(\mid p, \forall x \phi_{1}\right), B \rrbracket, \omega\right\rangle$ iff for every $c \in \mathcal{D}, \mathcal{B P}_{\varphi}$ has an accepting run from $\left\langle\llbracket\left(p, \phi_{1}\right), B[x \leftarrow c] \rrbracket, \omega\right\rangle$ since if $\mathcal{D}=\left\{c_{1}, \ldots, c_{m}\right\}$, then, $B \in \operatorname{meet}_{\mathcal{D}}^{x}\left(B\left[x \leftarrow c_{1}\right], \ldots, B\left[x \leftarrow c_{m}\right]\right)$

- If $\phi=E X^{g} \phi_{1}$, then, for every $\omega \in \Gamma^{*},\langle p, \omega\rangle \vDash_{\lambda}^{B} \phi$ iff there exists an immediate successor $\left\langle p^{\prime}, \omega^{\prime}\right\rangle$ of $\langle p, \omega\rangle$ s.t. $\left\langle p^{\prime}, \omega^{\prime}\right\rangle \vDash_{\lambda}^{B} \phi_{1}$. This is ensured by the transition rules in $(\hbar 7)$ stating that $\mathcal{B P} \varphi$ has an accepting run from $\left\langle\llbracket\left(p, E X^{g} \phi_{1}\right), B \rrbracket, \omega\right\rangle$ iff there exists an immediate successor $\left\langle p^{\prime}, \omega^{\prime}\right\rangle$ of $\langle p, \omega\rangle$ s.t. $\mathcal{B P}_{\varphi}$ has an accepting run from $\left\langle\llbracket\left(p^{\prime}, \phi_{1}\right), B \rrbracket, \omega^{\prime}\right\rangle$. ( $\left.\hbar 8\right)$ is similar to $(\hbar 7)$.

- If $\phi=E\left[\phi_{1} U^{g} \phi_{2}\right]$, then, for every $\omega \in \Gamma^{*},\langle p, \omega\rangle \vDash_{\lambda}^{B} \phi$ iff $\langle p, \omega\rangle \vDash_{\lambda}^{B} \phi_{2}$ or $\left(\langle p, \omega\rangle \vDash_{\lambda}^{B} \phi_{1}\right.$ and there exists an immediate successor $\left\langle p^{\prime}, \omega^{\prime}\right\rangle$ of $\langle p, \omega\rangle$ s.t. $\left.\left\langle p^{\prime}, \omega^{\prime}\right\rangle \vDash_{\lambda}^{B} \phi\right)$. This is ensured by the transition rules in ( $\left.\hbar 11\right)$ stating that $\mathcal{B P} \mathcal{P}_{\varphi}$ has an accepting run from $\left\langle\llbracket\left(p, E\left[\phi_{1} U^{g} \phi_{2}\right]\right), B \rrbracket, \omega\right\rangle$ iff $\mathcal{B P} \varphi$ has an accepting run from $\left\langle\llbracket\left(p, \phi_{2}\right), B \rrbracket, \omega\right\rangle$ (by the rules in $\left.(\hbar 11)(\mathrm{a})\right)$ or $(\mathcal{B P} \varphi$ has an accepting run from both $\left\langle\llbracket\left(\mid p, \phi_{1}\right), B \rrbracket, \omega\right\rangle$ and $\left\langle\llbracket\left(p^{\prime}, \phi\right), B \rrbracket, \omega^{\prime}\right\rangle$ where $\left\langle p^{\prime}, \omega^{\prime}\right\rangle$ is an immediate successor of $\langle p, \omega\rangle$ ) (by the rules in $\left.(\boldsymbol{\hbar} 11)(\mathrm{b})\right)$. ( $\hbar 13$ ) is similar to $(\hbar 11)$.

- If $\phi=E\left[\phi_{1} R^{g} \phi_{2}\right]$, then, for every $\omega \in \Gamma^{*},\langle p, \omega\rangle \vDash_{\lambda}^{B} \phi$ iff $\left(\langle p, \omega\rangle \vDash_{\lambda}^{B} \phi_{2}\right.$ and $\left.\langle p, \omega\rangle \vDash_{\lambda}^{B} \phi_{1}\right)$ or $\left(\langle p, \omega\rangle \vDash_{\lambda}^{B} \phi_{2}\right.$ and there exists an immediate successor $\left\langle p^{\prime}, \omega^{\prime}\right\rangle$ of $\langle p, \omega\rangle$ s.t. $\left.\left\langle p^{\prime}, \omega^{\prime}\right\rangle \vDash_{\lambda}^{B} \phi\right)$. This is ensured by the transition rules in $(\hbar 15)$ stating that $\mathcal{B P} \mathcal{P}_{\varphi}$ has an accepting run from $\left\langle\llbracket\left(\mid p, E\left[\phi_{1} R^{g} \phi_{2}\right]\right), B \rrbracket, \omega\right\rangle$ iff $\mathcal{B P}_{\varphi}$ has an accepting run from both $\left\langle\llbracket\left(p, \phi_{2}\right), B \rrbracket, \omega\right\rangle$ and $\left\langle\llbracket\left(\mid p, \phi_{1}\right), B \rrbracket, \omega\right\rangle$ (by the rules in $(\hbar \mathbf{1 5})(\mathrm{a}))$; or $\mathcal{B P} \mathcal{P}_{\varphi}$ has an accepting run from both $\left\langle\llbracket\left(p, \phi_{2}\right), B \rrbracket, \omega\right\rangle$ and $\left.\llbracket\left(p^{\prime}, \phi\right\rangle, B \rrbracket, \omega^{\prime}\right\rangle$ where $\left\langle p^{\prime}, \omega^{\prime}\right\rangle$ is an immediate successor of $\langle p, \omega\rangle$ (by the rules in $(\hbar 15)(\mathrm{b}))$. In addition, for $R^{g}$ formulas, the stop condition is not required, i.e, for a formula $\phi_{1} R^{g} \phi_{2}$ that is applied to a specific run, we don't require that $\phi_{1}$ must eventually hold. To ensure that the runs on which $\phi_{2}$ always holds are accepted, we add $\llbracket(p, \phi), B \rrbracket$ to the Büchi accepting condition $F$ (via the subset $F_{3}$ of $F$ ). ( $\left.\hbar 16\right)$ is similar to $(\hbar 15)$.

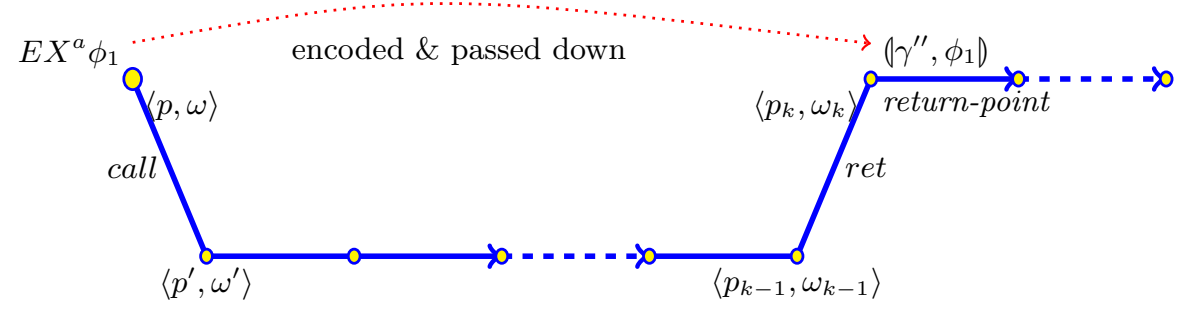

Fig. 2: $\langle p, \omega\rangle \Rightarrow_{\mathcal{P}}\left\langle p^{\prime}, \omega^{\prime}\right\rangle$ corresponds to a call statement

- If $\phi=E X^{a} \phi_{1}$, then, for every $\omega \in \Gamma^{*},\langle p, \omega\rangle \vDash_{\lambda}^{B} \phi$ iff there exists an abstractsuccessor $\left\langle p_{k}, \omega_{k}\right\rangle$ of $\langle p, \omega\rangle$ s.t. $\left\langle p_{k}, \omega_{k}\right\rangle \vDash_{\lambda}^{B} \phi_{1}$ (A1). Let $\pi \in \operatorname{Traces}(\langle p, \omega\rangle)$ be a run starting from $\langle p, \omega\rangle$ on which $\left\langle p_{k}, \omega_{k}\right\rangle$ is the abstract-successor of $\langle p, \omega\rangle$. Over $\pi$, let $\left\langle p^{\prime}, \omega^{\prime}\right\rangle$ be the immediate successor of $\langle p, \omega\rangle$. In what follows, we explain how we can ensure this. 
1. Firstly, we show that for every abstract-successor $\left\langle p_{k}, \omega_{k}\right\rangle \neq \perp$ of $\langle p, \omega\rangle$, $\left\langle\llbracket\left(p, E X^{a} \phi_{1}\right), B \rrbracket, \omega\right\rangle \Rightarrow_{\mathcal{B P}_{\varphi}}\left\langle\llbracket\left(\mid p_{k}, \phi_{1}\right), B \rrbracket, \omega_{k}\right\rangle$ where $B \in \mathcal{B}$. There are two possibilities:

- If $\langle p, \omega\rangle \Rightarrow_{\mathcal{P}}\left\langle p^{\prime}, \omega^{\prime}\right\rangle$ corresponds to a call statement. Let us consider Figure 2 to explain this case. $\langle\llbracket(\mid p, \phi), B \rrbracket, \omega\rangle \Rightarrow_{\mathcal{B} \mathcal{P}_{\varphi}}\left\langle\llbracket\left(p_{k}, \phi_{1}\right), B \rrbracket, \omega_{k}\right\rangle$ is ensured by the rules in $(\hbar 9)(\mathrm{a})$, the rules in $(\boldsymbol{\hbar 2 1})$ and the rules in $(\hbar 19)$ as follows: rules in $(\hbar 9)\left(\right.$ a) allow to record $\phi_{1}$ in the return point of the call, rules in $(\boldsymbol{\hbar 2 1})$ allow to mimic the run of the PDS $\mathcal{P}$ and rules in ( $\hbar 19)$ allow to extract and put back $\phi_{1}$ when the returnpoint is reached. In what follows, we show in more details how this works: Let $\langle p, \gamma\rangle \stackrel{\text { call }}{\longrightarrow}\left\langle p^{\prime}, \gamma^{\prime} \gamma^{\prime \prime}\right\rangle$ be the rule associated with the transition $\langle p, \omega\rangle \Rightarrow_{\mathcal{P}}\left\langle p^{\prime}, \omega^{\prime}\right\rangle$, then we have $\omega=\gamma \omega^{\prime \prime}$ and $\omega^{\prime}=\gamma^{\prime} \gamma^{\prime \prime} \omega^{\prime \prime}$. Let $\left\langle p_{k-1}, \omega_{k-1}\right\rangle \Rightarrow_{\mathcal{P}}\left\langle p_{k}, \omega_{k}\right\rangle$ be the transition that corresponds to the ret statement of this call on $\pi$. Let then $\left\langle p_{k-1}, \beta\right\rangle \stackrel{\text { ret }}{\longrightarrow}\left\langle p_{k}, \epsilon\right\rangle \in \Delta$ be the corresponding return rule. Then, we have necessarily $\omega_{k-1}=\beta \gamma^{\prime \prime} \omega^{\prime \prime}$, since as explained in Section $2, \gamma^{\prime \prime}$ is the return address of the call. After applying this rule, $\omega_{k}=\gamma^{\prime \prime} \omega^{\prime \prime}$. In other words, $\gamma^{\prime \prime}$ will be the topmost stack symbol at the corresponding return point of the call. So, in order to ensure that $\langle\llbracket(p, \phi), B \rrbracket, \omega\rangle \Rightarrow_{\mathcal{B P}_{\varphi}}\left\langle\llbracket\left(\mid p_{k}, \phi_{1}\right), B \rrbracket, \omega_{k}\right\rangle$, we proceed as follows: At the call $\langle p, \gamma\rangle \stackrel{\text { call }}{\longrightarrow}\left\langle p^{\prime}, \gamma^{\prime} \gamma^{\prime \prime}\right\rangle$, we encode the formula $\phi_{1}$ into $\gamma^{\prime \prime}$ by the rule in ( $\left.\hbar 9\right)$ (a) stating that $\left\langle\left(p, E X^{a} \phi_{1}\right), \gamma\right\rangle \stackrel{i d}{\longleftrightarrow}\left\langle p^{\prime}, \gamma^{\prime}\left(\gamma^{\prime \prime}, \phi_{1} D\right\rangle\right.$ $\in \Delta^{\prime}$. This allows to record $\phi_{1}$ in the corresponding return point of the stack. After that, the rules in $(\boldsymbol{\hbar 2 1})$ allow $\mathcal{B P}_{\varphi}$ to mimic the run $\pi$ of $\mathcal{P}$ from $\left\langle p^{\prime}, \omega^{\prime}\right\rangle$ till the corresponding return-point of this call, where $\left(\gamma^{\prime \prime}, \phi_{1}\right)$ is the topmost stack symbol. More specifically, the following sequence of $\mathcal{P}:\left\langle p^{\prime}, \gamma^{\prime} \gamma^{\prime \prime} \omega^{\prime \prime}\right\rangle \stackrel{*}{\Rightarrow} \mathcal{P}\left\langle p_{k-1}, \beta \gamma^{\prime \prime} \omega^{\prime \prime}\right\rangle \stackrel{*^{*}}{\Rightarrow} \mathcal{P}\left\langle p_{k}, \gamma^{\prime \prime} \omega^{\prime \prime}\right\rangle$ will be mimicked by the following sequence of $\mathcal{B P}_{\varphi}:\left\langle\llbracket p^{\prime}, B \rrbracket, \gamma^{\prime}\left(\gamma^{\prime \prime}, \phi_{1}\right) \omega^{\prime \prime}\right\rangle$ $\Rightarrow_{\mathcal{B P}_{\varphi}}\left\langle\llbracket p_{k-1}, B \rrbracket, \beta\left(\gamma^{\prime \prime}, \phi_{1}\right) \omega^{\prime \prime}\right\rangle \Rightarrow_{\mathcal{B P}_{\varphi}}\left\langle\llbracket p_{k}, B \rrbracket,\left(\gamma^{\prime \prime}, \phi_{1}\right) \omega^{\prime \prime}\right\rangle$ using the rules of $(\boldsymbol{\hbar 2 1})$. At the return-point, we extract $\phi_{1}$ from the stack and encode it into $p_{k}$ by adding the transition rules in $(\hbar 19)\left\langle p_{k},\left(\gamma^{\prime \prime}, \phi_{1}\right)\right\rangle \stackrel{i d}{\hookrightarrow}$ $\left\langle\left(p_{k}, \phi_{1}\right), \gamma^{\prime \prime}\right\rangle$. Therefore, we obtain that $\langle\llbracket(\mid p, \phi), B \rrbracket, \omega\rangle \Rightarrow_{\mathcal{B} \mathcal{P}_{\varphi}}\left\langle\llbracket\left(\mid p_{k}, \phi_{1}\right), B \rrbracket, \omega_{k}\right\rangle$. The property holds for this case.

- If $\langle p, \omega\rangle \Rightarrow_{\mathcal{P}}\left\langle p^{\prime}, \omega^{\prime}\right\rangle$ corresponds to a simple statement. Then, the abstract successor of $\langle p, \omega\rangle$ is its immediate successor $\left\langle p^{\prime}, \omega^{\prime}\right\rangle$. Thus, we get that $\left\langle p_{k}, \omega_{k}\right\rangle=\left\langle p^{\prime}, \omega^{\prime}\right\rangle$. From the transition rules ( $\left.\hbar 9\right)(\mathrm{b})$, we get that $\left\langle\llbracket\left(p, E X^{a} \phi_{1}\right), B \rrbracket, \omega\right\rangle \Rightarrow_{\mathcal{B} \mathcal{P}_{\varphi}}\left\langle\llbracket\left(p^{\prime}, \phi_{1}\right), B \rrbracket, \omega^{\prime}\right\rangle$. Therefore, $\left\langle\llbracket\left(\mid p, E X^{a} \phi_{1}\right), B \rrbracket, \omega\right\rangle$ $\Rightarrow_{\mathcal{B P}}\left\langle\llbracket \llbracket\left(p_{k}, \phi_{1}\right), B \rrbracket, \omega_{k}\right\rangle$. The property holds for this case.

2. Now, let us consider the case where $\left\langle p_{k}, \omega_{k}\right\rangle$, the abstract successor of $\langle p, \omega\rangle$, is $\perp$. This case occurs when $\langle p, \omega\rangle \Rightarrow_{\mathcal{P}}\left\langle p^{\prime}, \omega^{\prime}\right\rangle$ corresponds to a return statement. Then, one abstract successor of $\langle p, \omega\rangle$ is $\perp$. Note that $\perp$ does not satisfy any formula, i.e., $\perp$ does not satisfy $\phi_{1}$. Therefore, from $\left\langle\llbracket\left(\mid p, E X^{a} \phi_{1}\right), B \rrbracket, \omega\right\rangle$, we need to ensure that the path of $\mathcal{B P} \varphi$ reflecting the possibility in (A1) that $\left\langle p_{k}, \omega_{k}\right\rangle \vDash_{\lambda}^{B} \phi_{1}$ is not accepted. To do this, we exploit additional trap configurations. We use $p_{\perp}$ and $\gamma_{\perp}$ as trap control location and trap stack symbol to obtain these trap configurations. To be more specific, let $\langle p, \gamma\rangle \stackrel{\text { ret }}{\longrightarrow}\left\langle p^{\prime}, \epsilon\right\rangle$ be the rule associated with the transition $\langle p, \omega\rangle \Rightarrow_{\mathcal{P}}\left\langle p^{\prime}, \omega^{\prime}\right\rangle$, then we have $\omega=\gamma \omega^{\prime \prime}$ and $\omega^{\prime}=\omega^{\prime \prime}$. We add the transition rule in ( $\left.\hbar 9\right)(\mathrm{c})$ 
to allow $\left\langle\llbracket\left(p, E X^{a} \phi_{1}\right), B \rrbracket, \omega\right\rangle \Rightarrow_{\mathcal{B P}}\left\langle\llbracket p_{\perp}, B \rrbracket, \gamma_{\perp} \omega^{\prime \prime}\right\rangle$. Since a run of $\mathcal{B P} \mathcal{P}_{\varphi}$ includes only infinite paths, we equip these trap configurations with self-loops by the transition rules in ( $\mathbf{\hbar 2 0})$, i.e., $\left\langle\llbracket p_{\perp}, B \rrbracket, \gamma_{\perp} \omega^{\prime \prime}\right\rangle \Rightarrow_{\mathcal{B P}}\left\langle\llbracket p_{\perp}, B \rrbracket, \gamma_{\perp} \omega^{\prime \prime}\right\rangle$. As a result, we obtain a corresponding path in $\mathcal{B P}_{\varphi}:\left\langle\llbracket\left(\mid p, E X^{a} \phi_{1}\right), B \rrbracket, \omega\right\rangle \Rightarrow_{\mathcal{B} \mathcal{P}_{\varphi}}$ $\left\langle\llbracket p_{\perp}, B \rrbracket, \gamma_{\perp} \omega^{\prime \prime}\right\rangle \Rightarrow_{\mathcal{B} \mathcal{P}_{\varphi}}\left\langle\llbracket p_{\perp}, B \rrbracket, \gamma_{\perp} \omega^{\prime \prime}\right\rangle$. Note that this path is not accepted by $\mathcal{B P} \mathcal{P}_{\varphi}$ because $\llbracket p_{\perp}, B \rrbracket \notin F$.

In summary, for every abstract-successor $\left\langle p_{k}, \omega_{k}\right\rangle$ of $\langle p, \omega\rangle$, if $\left\langle p_{k}, \omega_{k}\right\rangle \neq \perp$, then, $\left\langle\llbracket\left(p, E X^{a} \phi_{1}\right), B \rrbracket, \omega\right\rangle \Rightarrow{ }_{\mathcal{B P}}\left\langle\llbracket \llbracket\left(p_{k}, \phi_{1}\right), B \rrbracket, \omega_{k}\right\rangle$; otherwise $\left\langle\llbracket\left(p, E X^{a} \phi_{1}\right), B \rrbracket, \omega\right\rangle \Rightarrow_{\mathcal{B} \mathcal{P}_{\varphi}}\left\langle\llbracket p_{\perp}, B \rrbracket, \gamma_{\perp} \omega^{\prime \prime}\right\rangle \Rightarrow_{\mathcal{B} \mathcal{P}_{\varphi}}\left\langle\llbracket p_{\perp}, B \rrbracket, \gamma_{\perp} \omega^{\prime \prime}\right\rangle$ which is not accepted by $\mathcal{B P} \mathcal{P}_{\varphi}$. Therefore, (A1) is ensured by the transition rules in ( $\hbar 9)$ stating that $\mathcal{B P} \varphi$ has an accepting run from $\left\langle\llbracket\left(\mid p, E X^{a} \phi_{1}\right), B \rrbracket, \omega\right\rangle$ iff there exists an abstract successor $\left\langle p_{k}, \omega_{k}\right\rangle$ of $\langle p, \omega\rangle$ s.t. $\mathcal{B P} \varphi$ has an accepting run from $\left\langle\llbracket\left(p_{k}, \phi_{1}\right), B \rrbracket, \omega_{k}\right\rangle$.

- If $\phi=A X^{a} \phi_{1}$ : this case is ensured by the transition rules in ( $\left.\hbar 10\right)$ together

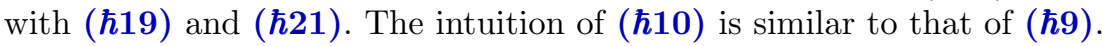

- If $\phi=E\left[\phi_{1} U^{a} \phi_{2}\right]$, then, for every $\omega \in \Gamma^{*},\langle p, \omega\rangle \vDash_{\lambda}^{B} \phi$ iff $\langle p, \omega\rangle \vDash_{\lambda}^{B} \phi_{2}$ or $\left(\langle p, \omega\rangle \vDash_{\lambda}^{B} \phi_{1}\right.$ and there exists an abstract successor $\left\langle p_{k}, \omega_{k}\right\rangle$ of $\langle p, \omega\rangle$ s.t. $\left.\left\langle p_{k}, \omega_{k}\right\rangle \vDash_{\lambda}^{B} \phi\right) \quad(\mathrm{A} 2)$. Let $\pi \in \operatorname{Traces}(\langle p, \omega\rangle)$ be a run starting from $\langle p, \omega\rangle$ on which $\left\langle p_{k}, \omega_{k}\right\rangle$ is the abstract-successor of $\langle p, \omega\rangle$. Over $\pi$, let $\left\langle p^{\prime}, \omega^{\prime}\right\rangle$ be the immediate successor of $\langle p, \omega\rangle$.

1. Firstly, we show that for every abstract-successor $\left\langle p_{k}, \omega_{k}\right\rangle \neq \perp$ of $\langle p, \omega\rangle$, $\langle\llbracket(p, \phi), B \rrbracket, \omega\rangle \Rightarrow_{\mathcal{B P} \varphi}\left\{\left\langle\llbracket\left(\mid p, \phi_{1}\right), B \rrbracket, \omega\right\rangle,\left\langle\llbracket\left(\mid p_{k}, \phi\right), B \rrbracket, \omega_{k}\right\rangle\right\}$ where $B \in \mathcal{B}$. There are two possibilities:

- If $\langle p, \omega\rangle \Rightarrow_{\mathcal{P}}\left\langle p^{\prime}, \omega^{\prime}\right\rangle$ corresponds to a call statement. From the rules in $(\hbar 12)(\mathrm{b})$, we get that $\langle\llbracket(\mid p, \phi), B \rrbracket, \omega\rangle \Rightarrow_{\mathcal{B} \mathcal{P}_{\varphi}}\left\{\left\langle\llbracket\left(\mid p, \phi_{1}\right), B \rrbracket, \omega\right\rangle,\left\langle p^{\prime}, \omega^{\prime}\right\rangle\right\}$ where $\left\langle p^{\prime}, \omega^{\prime}\right\rangle$ is the immediate successor of $\langle p, \omega\rangle$. Thus, to ensure that $\langle\llbracket(p, \phi), B \rrbracket, \omega\rangle \Rightarrow_{\mathcal{B P}_{\varphi}}\left\{\left\langle\llbracket\left(\mid p, \phi_{1}\right), B \rrbracket, \omega\right\rangle,\left\langle\llbracket\left(p_{k}, \phi\right), B \rrbracket, \omega_{k}\right\rangle\right\}$, we only need to ensure that $\left\langle p^{\prime}, \omega^{\prime}\right\rangle \Rightarrow_{\mathcal{B P}_{\varphi}}\left\langle\llbracket\left(p_{k}, \phi\right), B \rrbracket, \omega_{k}\right\rangle$. As for the case $\phi=$ $E X^{a} \phi_{1},\left\langle p^{\prime}, \omega^{\prime}\right\rangle \Rightarrow_{\mathcal{B P}}\left\langle\llbracket\left(p_{k}, \phi\right), B \rrbracket, \omega_{k}\right\rangle$ is ensured by the rules in ( $\left.\hbar 21\right)$ and the rules in $(\boldsymbol{\hbar 1 9})$ : rules in $(\boldsymbol{\hbar 2 1})$ allow to mimic the run of the PDS $\mathcal{P}$ before the return and rules in $(\hbar 19)$ allow to extract and put back $\phi_{1}$ when the return-point is reached.

- If $\langle p, \omega\rangle \Rightarrow_{\mathcal{P}}\left\langle p^{\prime}, \omega^{\prime}\right\rangle$ corresponds to a simple statement. Then, the abstract successor of $\langle p, \omega\rangle$ is its immediate successor $\left\langle p^{\prime}, \omega^{\prime}\right\rangle$. Thus, we

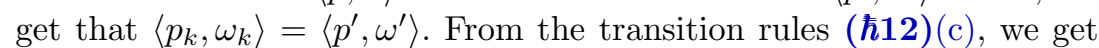
that $\left\langle\llbracket\left(\mid p, E\left[\phi_{1} U^{a} \phi_{2}\right]\right), B \rrbracket, \omega\right\rangle \Rightarrow_{\mathcal{B P}_{\varphi}}\left\{\left\langle\llbracket\left(\mid p, \phi_{1}\right), B \rrbracket, \omega\right\rangle,\left\langle\llbracket\left(\mid p^{\prime}, \phi\right), B \rrbracket, \omega^{\prime}\right\rangle\right\}$. Therefore, $\left\langle\llbracket\left(\mid p, E\left[\phi_{1} U^{a} \phi_{2}\right]\right), B \rrbracket, \omega\right\rangle \Rightarrow_{\mathcal{B} \mathcal{P}_{\varphi}}\left\{\left\langle\llbracket\left(\mid p, \phi_{1}\right), B \rrbracket, \omega\right\rangle,\left\langle\llbracket\left(p_{k}, \phi\right), B \rrbracket, \omega_{k}\right\rangle\right\}$. In other words, $\mathcal{B} \mathcal{P}_{\varphi}$ has an accepting run from both $\left\langle\llbracket\left(p, \phi_{1}\right), B \rrbracket, \omega\right\rangle$ and $\left\langle\llbracket\left(p_{k}, \phi\right), B \rrbracket, \omega_{k}\right\rangle$ where $\left\langle p_{k}, \omega_{k}\right\rangle$ is an abstract successor of $\langle p, \omega\rangle$. The property holds for this case.

2. Now, let us consider the case where $\left\langle p_{k}, \omega_{k}\right\rangle=\perp$. As explained previously, this case occurs when $\langle p, \omega\rangle \Rightarrow_{\mathcal{P}}\left\langle p^{\prime}, \omega^{\prime}\right\rangle$ corresponds to a return statement. Then, the abstract successor of $\langle p, \omega\rangle$ is $\perp$. Note that $\perp$ does not satisfy any formula, i.e., $\perp$ does not satisfy $\phi$. Therefore, from $\left\langle\llbracket\left(\left\langle p, E\left[\phi_{1} U^{a} \phi_{2}\right]\right), B \rrbracket, \omega\right\rangle\right.$, we need to ensure that the path reflecting the possibility in (A2) that $\left(\langle p, \omega\rangle \vDash_{\lambda}^{B} \phi_{1}\right.$ and $\left.\left\langle p_{k}, \omega_{k}\right\rangle \vDash_{\lambda}^{B} \phi\right)$ is not accepted by $\mathcal{B} \mathcal{P}_{\varphi}$. This is ensured as for the case $\phi=E X^{a} \phi_{1}$ by the transition rules in $(\hbar 12)(\mathrm{d})$. 
In summary, for every abstract-successor $\left\langle p_{k}, \omega_{k}\right\rangle$ of $\langle p, \omega\rangle$, if $\left\langle p_{k}, \omega_{k}\right\rangle \neq \perp$, then, $\left\langle\llbracket \llbracket p, E\left[\phi_{1} U^{a} \phi_{2} \rrbracket\right), B \rrbracket, \omega\right\rangle \Rightarrow_{\mathcal{B P}_{\varphi}}\left\{\left\langle\llbracket \mid p, \phi_{1} \emptyset, B \rrbracket, \omega\right\rangle,\left\langle\llbracket\left(\mid p_{k}, E\left[\phi_{1} U^{a} \phi_{2}\right]\right), B \rrbracket, \omega_{k}\right\rangle\right\} ;$ otherwise $\left\langle\llbracket\left(p, E\left[\phi_{1} U^{a} \phi_{2} \rrbracket\right), B \rrbracket, \omega\right\rangle \Rightarrow_{\mathcal{B} \mathcal{P}_{\varphi}}\left\langle\llbracket p_{\perp}, B \rrbracket, \gamma_{\perp} \omega^{\prime \prime}\right\rangle \Rightarrow_{\mathcal{B} \mathcal{P}_{\varphi}}\left\langle\llbracket p_{\perp}, B \rrbracket, \gamma_{\perp} \omega^{\prime \prime}\right\rangle\right.$ which is not accepted by $\mathcal{B P} \varphi$. Therefore, (A2) is ensured by the transition rules in ( $\hbar 12)$ stating that $\mathcal{B P} \varphi$ has an accepting run from $\left\langle\llbracket\left(\mid p, E\left[\phi_{1} U^{a} \phi_{2}\right]\right), B \rrbracket, \omega\right\rangle$ iff $\mathcal{B} \mathcal{P}_{\varphi}$ has an accepting run from $\left\langle\llbracket \mid p, \phi_{2} \rrbracket, B \rrbracket, \omega\right\rangle ;$ or $\mathcal{B} \mathcal{P}_{\varphi}$ has an accepting run from both $\left\langle\llbracket \mid p, \phi_{1} \emptyset, B \rrbracket, \omega\right\rangle$ and $\left\langle\llbracket \mid p_{k}, E\left[\phi_{1} U^{a} \phi_{2} \rrbracket\right), B \rrbracket, \omega_{k}\right\rangle$ where $\left\langle p_{k}, \omega_{k}\right\rangle$ is an abstract successor of $\langle p, \omega\rangle$.

- The intuition behind the rules corresponding to the cases $\phi=A\left[\phi_{1} U^{a} \phi_{2}\right]$, $\phi=A\left[\phi_{1} R^{a} \phi_{2}\right]$ are similar to the previous case.

- If $\phi=e(e \in \mathcal{V})$. Given $p \in P, e \in \mathcal{V}, \omega \in \Gamma^{*}$, we get that the SABPDS $\mathcal{B} \mathcal{P}_{\varphi}$ should accept $\langle\llbracket(p, e\rangle, B \rrbracket, \omega\rangle$ iff $(\langle p, \omega\rangle, B) \in L\left(M_{e}\right)$. To check whether $(\langle p, \omega\rangle, B) \in L\left(M_{e}\right)$, we let $\mathcal{B} \mathcal{P}_{\varphi}$ go to state $s_{e}$, the initial state corresponding to $p$ in $M_{e}$ by adding rules in ( $\left.\hbar 22\right)$; and then, from this state, we will check whether $\omega$ is accepted by $M_{e}$ under $B$. This is ensured by the transition rules in ( $(\mathbf{2 4})$ and $(\boldsymbol{\hbar 2 5})$. ( $(\mathbf{2 4})$ lets $\mathcal{B P} \mathcal{P}_{\varphi}$ mimic a run of $M_{e}$ on $\omega$ under $B$, which includes three possibilities:

- if $\mathcal{B P} \varphi$ is in a state $\llbracket q, B \rrbracket$ with $\gamma$ on the top of the stack where $\gamma \in \Gamma$, and if $q \stackrel{\gamma}{\rightarrow}\left\{q_{1}, \ldots, q_{n}\right\}$ is a transition rule in $M_{e}$, then, $\mathcal{B P} \varphi$ will move to states $\llbracket q_{1}, B \rrbracket, \ldots, \llbracket q_{n}, B \rrbracket$ and pop $\gamma$ from its stack. Note that popping $\gamma$ allows us to check the rest of the word. This is ensured by the rules corresponding to $(\boldsymbol{\hbar 2 4})(\mathrm{a})$. Then function equal ensures that all these environments are the same.

- if $\mathcal{B P} \varphi$ is in a state $\llbracket q, B \rrbracket$ with $\gamma$ on the top of the stack, and if $q \stackrel{x}{\rightarrow}$ $\left\{q_{1}, \ldots, q_{n}\right\}$ is a transition rule in $M_{e}$ where $x \in \mathcal{X}$, then, $\mathcal{B P}_{\varphi}$ can mimic a run of $M_{e}$ under $B$ iff $B(x)=\gamma$. If this condition is guaranteed, $\mathcal{B P} \varphi$ will move to states $\llbracket q_{1}, B \rrbracket, \ldots, \llbracket q_{n}, B \rrbracket$ and pop $\gamma$ from its stack. Again, popping $\gamma$ allows us to check the rest of the word. This is ensured by the rules corresponding to $(\hbar 24)(b)$. Then function $j o i n_{\gamma}^{x}$ ensures that all these environments are the same $B$ and $B(x)=\gamma$.

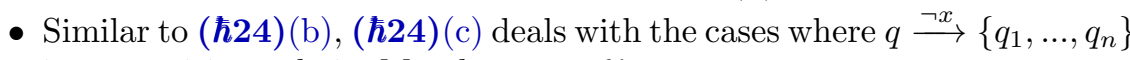
is a transition rule in $M_{e}$ where $x \in \mathcal{X}$.

In each VA $M_{e}$, a configuration is accepted if the run with the word $\omega$ reaches a final state in $F_{e}$; i.e., if $\mathcal{B P} \mathcal{P}_{\varphi}$ reaches a state $q \in F_{e}$ with an empty stack, i.e., with a stack containing the bottom stack symbol $\sharp$. Thus, we should add $F_{e} \times \mathcal{B}$ as a set of accepting control locations in $\mathcal{B P}_{\varphi}$. This is why $F_{4}$ is a set of accepting control locations. In addition, since $\mathcal{B P} \varphi$ only recognizes infinite paths, ( $\hbar 25)$ adds a loop on every configuration $\langle\llbracket q, B \rrbracket, \sharp\rangle$ where $q \in F_{e}$.

- If $\phi=\neg e(e \in \mathcal{V})$. This case is ensured by the transition rules in ( $\hbar 23)$, $(\hbar 24)$ and $(\boldsymbol{\hbar 2 5})$. The intuition behind this case is similar to the case $\phi=e$.

We can show that:

Theorem 4. Given a PDS $\mathcal{P}=(P, \Gamma, \Delta)$, a set of atomic propositions $A P$, a labelling function $\lambda: A P_{\mathcal{D}} \rightarrow 2^{P}$ and a SBPCARET formula $\varphi$, we can compute an $S A B P D S \mathcal{B P}_{\varphi}$ such that for every configuration $\langle p, \omega\rangle$, for every $B \in \mathcal{B}$, $\langle p, \omega\rangle \vDash_{\lambda}^{B} \varphi$ iff $\mathcal{B} \mathcal{P}_{\varphi}$ has an accepting run from the configuration $\langle\llbracket(p, \varphi\rangle, B \rrbracket, \omega\rangle$. 


\section{Conclusion}

In this paper, we present a new logic SBPCARET and show how it can precisely and succinctly specify malicious behaviors. We then propose an efficient algorithm for SBPCARET model-checking for PDSs. Our algorithm is based on reducing the model checking problem to the emptiness problem of Symbolic Alternating Büchi Pushdown Systems.

\section{References}

1. Rajeev Alur, Kousha Etessami, and P. Madhusudan. A temporal logic of nested calls and returns. In TACAS 2004.

2. J. Bergeron, M. Debbabi, J. Desharnais, M. M. Erhioui, Y. Lavoie, and N. Tawbi. Static detection of malicious code in executable programs. Int. J. of Req. Eng, 2001.

3. Mihai Christodorescu and Somesh Jha. Static analysis of executables to detect malicious patterns. In Proceedings of the 12th Conference on USENIX Security Symposium - Volume 12, SSYM'03, pages 12-12, Berkeley, CA, USA, 2003. USENIX Association.

4. Johannes Kinder, Stefan Katzenbeisser, Christian Schallhart, and Helmut Veith. Detecting malicious code by model checking. In Detection of Intrusions and Malware, and Vulnerability Assessment, Second International Conference, DIMVA 2005, Vienna, Austria, July 7-8, 2005, Proceedings, pages 174-187, 2005.

5. Arun Lakhotia, Eric Uday Kumar, and Michael Venable. A method for detecting obfuscated calls in malicious binaries. IEEE Trans. Software Eng., 31(11), 2005.

6. Huu-Vu Nguyen and Tayssir Touili. CARET model checking for malware detection. In SPIN 2017.

7. Huu-Vu Nguyen and Tayssir Touili. CARET model checking for pushdown systems. In $S A C 201 \%$.

8. Huu-Vu Nguyen and Tayssir Touili. Branching temporal logic of calls and returns for pushdown systems. In Integrated Formal Methods - 14th International Conference, IFM, 2018.

9. Stefan Schwoon. Model-Checking Pushdown Systems. Dissertation, Technische Universität München, München, 2002.

10. Fu Song and Tayssir Touili. Pushdown model checking for malware detection. In TACAS 2012.

11. Fu Song and Tayssir Touili. Efficient malware detection using model-checking. In FM 2012: Formal Methods - 18th International Symposium, 2012.

12. Fu Song and Tayssir Touili. LTL model-checking for malware detection. In TACAS 2013, 2013.

13. Fu Song and Tayssir Touili. Pommade: pushdown model-checking for malware detection. In SIGSOFT 2013, 2013. 